\title{
Present-day stress field changes along the Baikal rift and tectonic implications
}

\author{
Carole Petit, Jacques Déverchère, and Frédérique Houdry \\ Géosciences Azur EP125, Université Pierre et Marie Curie, Villefranche-sur-Mer, France
}

Vladimir A. Sankov and Valentina I. Melnikova

Institute of the Earth's Crust, Russian Academy of Sciences, Irkutsk, Russia

\author{
Damien Delvaux \\ Royal Museum for Central Africa, Tervuren, Belgium
}

\begin{abstract}
Intraplate extension, in a frame of a global compressional stress field, seems linked to local lithospheric perturbations (lithospheric thinning or thickening) able to modify the resulting state of stress [Zoback, 1992]. The Baikal Rift Zone (BRZ), Siberia, is located north of the India-Asia collision zone and exhibits no direct communication with any oceanic domain. It can thus be fully considered as an area of continental extension, dominated by the "global compressional intraplate stress field" resulting from plate driving forces. In order to address the problem of its dynamics and kinematics and their links with the India-Asia collision, a comprehensive stress tensor analysis is presented, based on 319 focal mechanisms of earthquakes located along the whole Baikal rift. The stress field is varying at different scales of observation: when looking at central Asia (several thousands kilometers), the maximum horizontal stress $S_{\text {Lmax }}$ directions remain rather constant (with a fan-shape geometry) when the tectonic regime goes from compressional (Himalayas) to extensional (Baikal). When observing the Baikal rift (about $1000 \mathrm{~km}$ long), clear variations of the stress regime are observed, from an extensional regime in the central part of the rift to wrench ones in its northern and southern ends. Finally, at the scale of $100 \mathrm{~km}$, systematic $S_{H \max }$ reorientations occur close to major rift faults. We thus infer that the interaction between collisional processes and inherited structures may have a strong influence on rift dynamics. We then use computed stress tensors to predict slip vectors on major rift faults. Deformation patterns show two distinct parts of the rift: the South Baikal Rift (SBR) is characterized by a constant trending (around $\mathrm{N} 100^{\circ} \mathrm{E}$ ) slip vector, meanwhile the North Baikal Rift (NBR) exhibits a complex block rotation behavior involving at least three crustal blocks. We propose to interpret these surficial structures and motions as the result of an interaction between the regional compression coming from the India-Asia collision and the geometry of the hardly deformable Siberian platform. This particular setting can explain most of the surficial deformation patterns, which suggest a largescale cracking of the lithosphere in the Baikal region. Other possible sources of stress could also be considered, like deep mantellic upwelling, or trench suction linked to the Pacific subduction.
\end{abstract}

Copyright 1996 by the American Geophysical Union.

Paper number $96 \mathrm{TC} 00624$ 0278-7407/96/96TC-00624\$12.00

\section{Introduction}

Relationships of the stress field to tectonics and structure of the upper lithosphere are complex and controlled both by local and regional factors. Stress field studies in active zones have widely developed within the last 10 years by means of in situ measurements, fault slip data and focal mechanisms. The World Stress Map Project [Zoback et al., 1989; Zoback, 1992] gathered informations on maximum horizontal stress orientations over the world, which were then compared to global models of plate motions. Although broad-scale (first-order) stress patterns appear to be the result of plate-driving forces [Bosworth et al., 1992; Richardson, 1992], it is shown that the addition of local secondorder stresses, due for example to lithospheric flexure or buoyancy forces, may strongly affect the resulting stress field [Zoback, 1992].

More specifically, data collected inside continental areas show that in most cases, this first-order stress field is compressional. Continental extensional dynamics thus involves the influence of second-order stresses. For example, buoyancy stresses related to the existence of lithospheric heterogeneities (crustal and lithospheric thinning or thickening) may be of the same order of magnitude as first-order stresses induced by plate motions [Zoback et al., 1989; Zoback, 1992] and predominate upon them. This observation implies that continental extension occurs at the place where two independent phenomena of similar magnitude are superimposed, the first one resulting from plate-driving forces and the second one from local lithospheric perturbations. However, it is not clear whether these perturbations are a cause or an effect of rifting dynamics.

This matter of intracontinental rifting and its relationships to neighboring compressional areas is of special interest in the Baikal Rift Zone (BRZ) where active extensional tectonics extends over $1500 \mathrm{~km}$, north of the India-Asia collision. The problem of the important positive relief associated with rifting has been partially addressed by gravity modelling, which shows the high effective elastic thickness of the lithosphere [Diament and Kogan, 1990; Ruppel et al., 1993; Burov et al., 1994]. Thus lithospheric flexure may be partly responsible for the elevated topography observed in the BRZ. Asthenospheric upwelling is also a possible agent of rifting process, although it seems small in the Northern Baikal Rift (NBR) [Burov et al., 1994; Petit and Déverchère, 1995]. Nevertheless, an asymmetric uplift of the asthenosphere seems located beneath the southern lake Baikal at 
depths up to $50 \mathrm{~km}$ [Gao et al, 1994]. Concerning vertical movements of the lithosphere in the BRZ, the predominance of one of these factors upon the other is thus not clearly elucidated.

The large extent of the Baikal rift system, as well as the diversity of its structural orientations, allow us to study the interaction of the stress field with active faults and their links with the Asian velocity field. Analogical experiments [Tapponnier et al,, 1982; Davy and Cobbold, 1988; Peltzer and Tapponnier, 1988] and horizontal kinematic models of central Asia [Avouac and Tapponnier, 1993] favor a direct link between the India-Asia collision and the existence of the BRZ. The particular S-shaped geometry of the rift, with en echelon disposition of faults and basins in the NBR, suggests that oblique extension resulting from extrusion processes may take a significant part in its opening. However, clear field evidence is scarce and concerns only Holocene cumulative displacements and recent Cenozoic microtectonic data [Houdry, 1994; D. Delvaux et al., Paleostress reconstructions and geodynamics of the Baikal region, Central Asia, II, Cenozoic tectonic stress and fault kinematics, submitted to Tectonophysics, 1996]. Instantaneous velocity field measurements by Global Positioning System (GPS) have been performed for the first time in the BRZ during the summer of 1994 [Petit et al., 1994] and will provide the first results in 1997. From the already available information, several questions are worth pointing out: what are the characteristics of the stress field in the Baikal region and which links can be drawn between it and the India-Asia collision? Is the direction of the maximum horizontal stress consistent with the velocity field within deforming Asia? How does the present-day rift deformation relate to the Asian strain field? Detailed imaging of the present-day stress field in the rift zone should allow us to shed light on these points and better constrain the dynamics and kinematics of the rift. The numerous data used here (319 focal mechanisms, see Plate 1) make the Baikal rift the bestdocumented active rift system in the world. In the present paper, we use this comprehensive data set of fault plane solutions in order to determine the stress field characteristics in different parts of the rift and try to correlate them with regional stress patterns of Asia. Using these results, and assuming that the slip vector on a given fault results from the combined effect of the local stress field and the fault geometry, we predict the displacement field on the basis of accurate field and satellite data on major rift faults, and infer a qualitative model of deformation of the rift area.

\section{From Individual Strain Observations to Regional Stress Field}

\section{Inversion Methods and Selection of Nodal Planes}

Several indicators may be used to determine the stress field, such as in situ measurements, which allow us to constrain the local tectonic stress in both orientation and magnitude [see e.g., Shamir et al., 1988; Cornet and Burlet, 1992; Rebaï et al., 1992], observation of tectonic structures like striated fault planes [see e.g., Angelier, 1979; Rebaï et al., 1992; Hippolyte et al., 1994], and focal mechanisms of earthquakes [see e.g., McKenzie, 1969; Michael, 1987; Hartse et al., 1994]. The use of fault plane solutions for stress inversion implies homogeneous data sets and the choice of the actual fault plane among the two proposed nodal planes [Michael, 1987; Horiuchi et al., 1995]. Its advantage is the abundance of data in seismic zones where other informations (i.e., microtectonic measurements) are scarce. Focal mechanisms give the slip vector on two possible fault planes, only one of which is the actual seismic fault. If the stress tensor is given and triaxial, only the actual fault plane will give a slip vector consistent with the stress tensor. If not, superimposition of compressional and tensional quadrants of several focal mechanisms in a given area will provide compressional and tensional zones where the maximal and minimal stress axes have to be searched [Angelier and Mechler, 1977]. This first estimation of the stress tensor is often enough to discriminate between the two nodal planes of each mechanism, and allows creation of a preferred set of planes representing seismic faults. Inversion methods are then used to minimize the deviation between observed and calculated striations, in order to refine the stress tensor determination [see e.g., Gephart and Forsyth, 1984; Carey-Gailhardis and Mercier, 1987; Rivera and Cisternas, 1990]. Nevertheless, the choice of the likely failure plane remains sometimes ambiguous and does not avoid, when possible, a comparison with strike and dip of active faults on the field.

In order to refine this discrimination, we compare in this study the results obtained by the use of two numerical methods [CareyGailhardis and Mercier, 1987; Delvaux et al., 1996]. Both algorithms involve three similar steps. The first step is the estimation of main stress axes directions by the right dihedra method of Angelier and Mechler [1977]. Then, applying this initial tensor to the whole data set, the selection of preferred fault planes is made using different criteria (see after). Finally, an inversion procedure (in a least squares sense) provides the new tensor, using the preferred set of data, by the adjustment of several possible parameters, among which are the slip deviation, that is the difference between calculated and observed slip vector orientation, used in both methods, and the friction coefficient, used in the Delvaux et al. [1996] routine.

To select the best-fitting fault plane, Carey-Gailhardis and Mercier [1987] use the following criteria: the deviation between calculated and observed slip vectors which must be less than $20^{\circ}$, and the $R$ ratio for each fault plane, where $R=\left(\sigma_{2}-\sigma_{1}\right) /\left(\sigma_{3}-\sigma_{1}\right)$, must range between 0 and 1 . When the stress tensor is well defined, these criteria are theoritically accurate enough to distinguish the fault plane from the auxiliary one. In the present case, additional information such as geological microtectonic data would help greatly to constrain this discrimination, but the uncertainties on hypocenter locations, as well as the high fracture density, do not allow association of nodal planes with wellknown fault tracks. Thus, in order to control this determination, we also use the program of Delvaux et al. [1996] which computes the internal friction coefficient $\mu$ for each fault plane and compares it with fracture criteria values described by the expression of the Mohr envelopes:

$$
\sigma_{s}=C O H+\mu \sigma_{n}
$$

Where $\sigma_{s}$ and $\sigma_{n}$ are the shear and normal stresses, respectively. $\mathrm{COH}$ describes the cohesion of the rock, and $\mu$ is the coefficient of internal friction, which is equal to $\tan (\Omega)$ where $\Omega$ is the angle of internal friction.

This describes the ability of the tensor to activate or not activate preexisting fractures: the fault plane is activated only if its position on the Mohr diagram is above the envelope. This criterion may be of delicate use because of the lack of knowledge on the mechanical and rheological state of the rocks. Zoback and 
Beroza [1991] have shown that near-lithostatic pore pressure may significantly reduce the resistance to fault motion. However, as reported later, comparing the preferred sets of planes selected with this parameter or with the slip deviation criteria allows us to give an estimate of the accuracy of stress tensor determination.

\section{Data Set: Selection and Uncertainties of Stress Inversion}

We use a total database of 319 focal mechanisms provided by different sources and recorded between 1950 and 1994 (Table 1). Magnitudes range between 2.8 and 7.8. Russian solutions represent the major part of the data set, involving 308 mechanisms, among which 161 are composite solutions. Thirteen other mechanisms come from waveform modelling made by Doser [1991a,b]. Finally, 11 mechanisms are Centroid Moment Tensor Solutions (CMTS) determined with the method of Dziewonski and Woodhouse [1983]. The geographical distribution of fault plane solutions is shown on Plate 1. Fourteen focal mechanisms of the Russian data set were also determined by other procedures: waveform modelling [Doser, 1991a,b] or CMT determinations (Table 1). In most cases, the discrepancies between these determinations are small: angular differences on $P$ and $T$ axis are less than $20^{\circ}$ for $85 \%$ of the solutions. We have generally considered that waveform modelling (especially CMT) solutions are more accurate, except for the Mondy earthquake of April 4, 1950, for which the only consistent solution is given by the first motion determination [Solonenko, 1977].

Single focal mechanisms represent 151 nonambiguous solutions of a comprehensive Russian database of 40 years of seismicity [Solonenko et al., 1993]. We applied here the procedure used by Déverchère et al. [1993] to select these reliable mechanisms. Composite solutions are numerous in our data set $(50 \%)$ and often subject of suspicion in seismological studies, mainly because they rely on the additional assumption that several nearby earthquakes depict the same fault motion. An ideal way to avoid this problem is to compute the stress tensor using only first motion readings, without previous fault plane determinations [Rivera and Cisternas, 1990]. Nevertheless, it has been demonstrated that earthquakes occurring in a wide area, on randomly oriented faults, may statistically help to constrain the principal stress axes [Xu et al., 1992]. In other words, when constructing composite fault plane solutions with a large number of data, the variations of $P$ and $T$ axes due to local fault geometries cancel each other. The resulting "best-fitting" $P$ and $T$ axes hence represent a good estimation of the maximal and minimal stresses, respectively. Stress tensor determinations by the superimposition of tensional and compressional dihedrons act in the same way. We thus believe that well-constrained composite solutions are reliable, and represent an important amount of information on the local stress field. Each composite solution selected in this study is made of nearby earthquakes grouped inside a $10 \times 10 \mathrm{~km}$ area, with compatible first $P$-wave arrivals (including an important amount of direct waves) at the same stations. Uncertainties on focal depths are ranging within 5 $10 \mathrm{~km}$ and do not significantly affect first motion distribution on the focal spheres. These conditions allow us to consider a group of similar shocks as if it was an individual event.

The data set has been geographically divided into 15 subareas (Figure 1) used for stress tensor determination. It is supposed that the size and position of the different subregions can influence each corresponding stress tensor. Indeed, it appears that resizing the subregions may slightly modify the computed stress tensor.
Actually, two simple alternatives arise: either the stress field changes continuously, and the result will be a representative average of the stress field in the selected area, if the latter is small enough compared to the scale of stress patterns; or stress field variations are strong and discrete, and the result will be associated with a variable amount of incompatible focal mechanisms if the chosen subregion is poorly positioned. An a posteriori control on rejected solutions is thus needed in order to insure the validity of the subsets. We made the choice to separate the data set taking into account the main active features for a given area: each subregion tends to include the entire length of main active faults (Figure 1) because stress tensors applied to each structural feature are supposed to be homogeneous. A last limitation when choosing the size of subregions is the data density. A percentage of selected solutions is presented in Table 2 , showing that about $85 \%$ of the focal mechanisms are compatible with the obtained tensors.

Finally, we performed a test on the validity of the data set (especially on composite solutions) in the Muyakan area (Figure 1). This region has been the subject of stress tensor determination by Déverchère et al. [1993]: first motions of 39 small magnitude (2.2-4.4) earthquakes recorded between 1977 and 1980 were used to determine accurate single fault plane solutions and a stable extensional stress tensor. In the present study, we use in the same area 43 focal mechanisms, among which 35 are composite solutions and 8 are single focal solutions coming from moderate magnitude (2.8-5.7) events. This region thus provides the opportunity to test the consistency of our data and to estimate the reliability of composite solutions. Table 3 compares our stress tensor determination to that of Déverchère et al. [1993]. Despite of the use of different samples, the computed stress tensors remain quite similar: horizontal stress directions are identical to within $4^{\circ}$, and resulting $R$ values are equal (Table 3 and Figure 2). Magnitude differences between both earthquake samples thus do not appear to affect the result of stress tensor inversion. Moreover, composite solutions seem to statistically constrain the stress field with rather good accuracy.

How far do uncertainties on data set (choice of fault planes) and on computing (minimization procedures) affect the final inversion? The Carey-Gailhardis and Mercier [1987] and the Delvaux et al. [1996] methods are compared in order to depict the stability of the obtained stress tensors (Figure 2). In most cases, these methods lead to the selection of similar sets of nodal planes (divergent choices are made for about $8 \%$ of the planes). They also lead to similar stress directions, especially for $\sigma_{2}$ and $\sigma_{3}$ whose orientations are better constrained because they are often nearly horizontal. Differences between these two determinations are thought to be a good estimate of uncertainties on stress computations, but do not represent actual errors, which also depend on the data density. Consequently, an average tensor solution is deduced from both solutions computed for each subset (Table 2). Differences between this average and one or the other solution are supposed to reflect computational uncertainties on dip and plunge of each axis and on $R$ value. A quality factor is assigned to each result, depending on the total number of data and on the number of solutions kept for the inversion (Table 2), in order to evaluate their relative reliability.

\section{Results of Stress Tensor Determination}

A stress tensor can be described either in terms of stress orientation, or in terms of tectonic regime. The latter is presented 
Table 1. List of the 319 Fault Plane Solutions Used in This Study, Grouped into 15 Subsets

\begin{tabular}{|c|c|c|c|c|c|c|c|}
\hline Date / Information & Latitude & Longitude & Strike & Dip & Rake & Magnitude & Origin \\
\hline \multicolumn{8}{|c|}{ Region: Bolnai } \\
\hline 630601 & 49.60 & 99.50 & 100 & 26 & 90 & 4.5 & Irkutsk \\
\hline 670607 & 49.50 & 97.20 & 60 & 58 & 130 & 5.0 & Novosibirsk \\
\hline 750821 & 49.63 & 97.38 & 240 & 71 & -29 & 4.5 & Irkutsk \\
\hline 750925 & 49.57 & 98.35 & 74 & 45 & 104 & 4.5 & Novosibirsk \\
\hline 770929 & 49.66 & 97.88 & 351 & 45 & 96 & 4.5 & Novosibirsk \\
\hline 781112 & 49.08 & 99.72 & 92 & 45 & 139 & 4.4 & Novosibirsk \\
\hline 791213 & 49.62 & 97.88 & 326 & 45 & -34 & 4.5 & Novosibirsk \\
\hline 830409 & 49.21 & 97.94 & 201 & 50 & -124 & 3.5 & Irkutsk \\
\hline 860407 & 49.47 & 98.90 & 28 & 50 & -98 & 4.5 & Irkutsk \\
\hline 861212 & 49.62 & 99.86 & 24 & 70 & 82 & 2.8 & Irkutsk \\
\hline 890203 & 49.61 & 97.41 & 111 & 41 & 108 & 4.5 & Novosibirsk \\
\hline 890515 & 49.72 & 97.86 & 225 & 45 & 94 & 4.5 & Novosibirsk \\
\hline Composite & 49.68 & 97.26 & 209 & 78 & -37 & & Irkutsk \\
\hline Composite & 49.54 & 97.15 & 62 & 86 & -97 & & Irkutsk \\
\hline Composite & 49.55 & 97.56 & 14 & 82 & -153 & & Irkutsk \\
\hline Composite & 49.45 & 97.19 & 271 & 54 & 87 & & Irkutsk \\
\hline Composite & 49.33 & 98.45 & 196 & 30 & 14 & & Irkutsk \\
\hline Composite & 49.46 & 98.46 & 14 & 53 & 74 & & Irkutsk \\
\hline Composite & 49.39 & 98.01 & 283 & 50 & 63 & & Irkutsk \\
\hline \multicolumn{8}{|c|}{ Region: Busingol } \\
\hline 660510 & 51.70 & 98.92 & 265 & 70 & 15 & 5.8 & Irkutsk \\
\hline 720527 & 51.37 & 98.05 & 61 & 45 & 146 & 4.5 & Novosibirsk \\
\hline 741129 & 51.79 & 98.47 & 137 & 45 & 157 & 5.2 & Novosibirsk \\
\hline 760401 & 51.06 & 98.03 & 148 & 80 & 166 & 5.5 & Irkutsk \\
\hline 780831 & 50.93 & 98.53 & 47 & 58 & -22 & 4.5 & Irkutsk \\
\hline 831120 & 51.31 & 98.38 & 78 & 67 & -53 & 4.5 & Novosibirsk \\
\hline Composite & 51.05 & 98.01 & 22 & 44 & -153 & 4.0 & Irkutsk \\
\hline Composite & 51.00 & 98.00 & 315 & 84 & 127 & & Irkutsk \\
\hline Composite & 51.06 & 98.19 & 69 & 56 & 69 & & Irkutsk \\
\hline Composite & 51.01 & 97.98 & 230 & 64 & 136 & & Irkutsk \\
\hline Composite & 51.00 & 98.22 & 254 & 62 & 42 & & Irkutsk \\
\hline Composite & 51.14 & 98.18 & 47 & 58 & -22 & & Irkutsk \\
\hline Composite & 51.12 & 97.96 & 236 & 82 & 21 & & Irkutsk \\
\hline Composite & 50.95 & 98.05 & 198 & 72 & 21 & & Irkutsk \\
\hline Composite & 51.83 & 98.35 & 86 & 87 & -10 & & Irkutsk \\
\hline Composite & 50.36 & 98.05 & 264 & 50 & 76 & & Irkutsk \\
\hline Composite & 51.75 & 98.33 & 107 & 70 & 48 & & Irkutsk \\
\hline \multicolumn{8}{|c|}{ Region: Khubsugul } \\
\hline 760401 & 50.62 & 100.22 & 293 & 48 & 72 & 4.5 & Irkutsk \\
\hline 850406 & 51.36 & 100.61 & 25 & 48 & -102 & 4.8 & Irkutsk \\
\hline 850824 & 51.20 & 100.40 & 0 & 44 & -126 & 4.0 & Irkutsk \\
\hline 870308 & 51.30 & 100.36 & 348 & 56 & -117 & 4.0 & Irkutsk \\
\hline Composite & 51.44 & 99.82 & 70 & 85 & -50 & & Irkutsk \\
\hline Composite & 51.74 & 100.35 & 240 & 20 & 28 & & Irkutsk \\
\hline Composite & 51.36 & 100.62 & 235 & 47 & -90 & & Irkutsk \\
\hline Composite & 51.69 & 100.83 & 325 & 52 & 94 & & Irkutsk \\
\hline \multicolumn{8}{|c|}{ Region: Transbaikal } \\
\hline $570206 *$ & 50.12 & 105.32 & 154 & 80 & -10 & 6.5 & Doser \\
\hline 580623 & 48.70 & 103.20 & 1 & 70 & 151 & 6.2 & Irkutsk \\
\hline 611009 & 51.50 & 104.80 & 10 & 56 & -150 & 3.5 & Irkutsk \\
\hline 630423 & 47.00 & 103.60 & 217 & 34 & 178 & 5.0 & Irkutsk \\
\hline 670105 & 48.10 & 102.90 & 13 & 88 & 171 & 7.8 & Irkutsk \\
\hline 670105 & 47.95 & 103.00 & 351 & 69 & 174 & 5.0 & Irkutsk \\
\hline 670107 & 48.00 & 103.00 & 13 & 41 & -150 & 5.0 & Irkutsk \\
\hline 670118 & 47.90 & 102.90 & 30 & 70 & 111 & 5.0 & Irkutsk \\
\hline 670120 & 48.10 & 103.10 & 348 & 62 & 170 & 7.0 & Irkutsk \\
\hline 670603 & 49.20 & 104.00 & 36 & 56 & 150 & 4.5 & Irkutsk \\
\hline 741218 & 48.35 & 103.15 & 255 & 86 & -38 & 5.4 & Irkutsk \\
\hline 840805 & 49.10 & 101.29 & 174 & 80 & 126 & 4.8 & Irkutsk \\
\hline 870301 & 49.78 & 102.45 & 52 & 40 & -100 & 5.2 & Irkutsk \\
\hline 890513 & 50.18 & 105.39 & 206 & 84 & 162 & 5.0 & Irkutsk \\
\hline
\end{tabular}


Table 1. (continued)

\begin{tabular}{|c|c|c|c|c|c|c|c|}
\hline Date / Information & Latitude & Longitude & Strike & Dip & Rake & Magnitude & Origin \\
\hline \multicolumn{8}{|c|}{ Region: Sayan } \\
\hline $500404^{*}$ & 51.80 & 101.00 & 282 & 24 & 90 & 7.0 & Irkutsk \\
\hline 620122 & 52.40 & 100.40 & 187 & 32 & 99 & 5.5 & Irkutsk \\
\hline 800723 & 52.32 & 102.63 & 80 & 62 & -123 & 4.5 & Irkutsk \\
\hline 811201 & 52.18 & 101.00 & 203 & 40 & -168 & 5.0 & Irkutsk \\
\hline Composite & 51.74 & 100.35 & 240 & 20 & 28 & & Irkutsk \\
\hline Composite & 51.69 & 100.83 & 325 & 52 & 94 & & Irkutsk \\
\hline Composite & 51.86 & 101.16 & 314 & 44 & 93 & & Irkutsk \\
\hline Composite & 51.84 & 101.04 & 269 & 36 & -95 & & Irkutsk \\
\hline Composite & 51.74 & 101.41 & 307 & 35 & 88 & & Irkutsk \\
\hline Composite & 51.78 & 101.40 & 315 & 52 & -40 & & Irkutsk \\
\hline Composite & 51.74 & 101.44 & 336 & 50 & -92 & & Irkutsk \\
\hline Composite & 51.71 & 101.38 & 32 & 40 & -121 & & Irkutsk \\
\hline Composite & 51.62 & 101.32 & 218 & 71 & -40 & & Irkutsk \\
\hline Composite & 51.66 & 101.75 & 225 & 34 & 31 & & Irkutsk \\
\hline Composite & 51.68 & 101.94 & 15 & 46 & -149 & & Irkutsk \\
\hline Composite & 51.78 & 101.92 & 289 & 54 & 75 & & Irkutsk \\
\hline Composite & 51.69 & 102.04 & 84 & 70 & -36 & & Irkutsk \\
\hline Composite & 51.71 & 102.21 & 16 & 35 & -114 & & Irkutsk \\
\hline Composite & 51.86 & 102.81 & 4 & 88 & -146 & & Irkutsk \\
\hline \multicolumn{8}{|c|}{ Region: Southern Baikal } \\
\hline $590829 *$ & 52.64 & 106.90 & 248 & 53 & -50 & 6.8 & Doser \\
\hline 600312 & 52.00 & 105.80 & 210 & 50 & -93 & 4.5 & Irkutsk \\
\hline 600519 & 52.10 & 105.70 & 210 & 50 & -93 & 4.0 & Irkutsk \\
\hline 610807 & 52.40 & 106.60 & 15 & 50 & -133 & 4.0 & Irkutsk \\
\hline 611009 & 51.50 & 104.80 & 10 & 56 & -150 & 3.5 & Irkutsk \\
\hline 630210 & 52.60 & 106.80 & 33 & 50 & -114 & 5.0 & Irkutsk \\
\hline 660830 & 51.69 & 104.49 & 255 & 62 & 26 & 5.5 & Irkutsk \\
\hline 670119 & 52.10 & 106.40 & 42 & 37 & -99 & 4.0 & Irkutsk \\
\hline 670211 & 52.09 & 106.46 & 218 & 67 & 168 & 5.3 & Doser \\
\hline 700328 & 52.20 & 105.92 & 82 & 54 & 4 & 5.5 & Irkutsk \\
\hline 700813 & 51.95 & 105.53 & 37 & 40 & -113 & 4.9 & Irkutsk \\
\hline 800206 & 51.74 & 105.14 & 217 & 80 & -95 & 4.9 & Irkutsk \\
\hline 800730 & 52.61 & 106.92 & 17 & 68 & -101 & 4.5 & Irkutsk \\
\hline 800926 & 51.86 & 105.33 & 68 & 18 & -106 & 4.5 & Irkutsk \\
\hline $810522^{*}$ & 52.05 & 106.32 & 18 & 18 & -137 & 5.4 & CMTS \\
\hline 820727 & 52.43 & 106.65 & 211 & 56 & -68 & 4.7 & Irkutsk \\
\hline 831124 & 52.99 & 106.95 & 220 & 80 & -114 & 4.5 & Irkutsk \\
\hline 850310 & 52.70 & 106.98 & 25 & 64 & -105 & 4.8 & Irkutsk \\
\hline 850325 & 52.27 & 106.43 & 317 & 40 & -137 & 4.5 & Irkutsk \\
\hline $850903^{*}$ & 52.85 & 106.87 & 343 & 82 & -167 & 4.4 & Doser \\
\hline 870329 & 52.19 & 106.20 & 58 & 40 & -55 & 4.0 & Irkutsk \\
\hline 870511 & 51.71 & 105.28 & 40 & 66 & -86 & 4.8 & Irkutsk \\
\hline $890513^{*}$ & 52.20 & 105.93 & 206 & 70 & -174 & 5.8 & CMTS \\
\hline Composite & 51.59 & 104.53 & 255 & 62 & -24 & & Irkutsk \\
\hline Composite & 51.79 & 105.23 & 40 & 30 & -110 & & Irkutsk \\
\hline Composite & 51.87 & 105.18 & 94 & 28 & -54 & & Irkutsk \\
\hline Composite & 52.00 & 105.59 & 37 & 40 & -112 & & Irkutsk \\
\hline Composite & 52.17 & 105.80 & 350 & 86 & 145 & & Irkutsk \\
\hline Composite & 52.17 & 105.83 & 171 & 54 & -121 & & Irkutsk \\
\hline Composite & 52.21 & 106.38 & 80 & 51 & -100 & & Irkutsk \\
\hline Composite & 52.02 & 106.32 & 66 & 52 & -48 & & Irkutsk \\
\hline Composite & 52.40 & 106.75 & 21 & 42 & -112 & & Irkutsk \\
\hline Composite & 52.56 & 106.97 & 44 & 49 & -96 & & Irkutsk \\
\hline Composite & 52.53 & 106.91 & 0 & 66 & -156 & & Irkutsk \\
\hline \multicolumn{8}{|c|}{ Region: Ceniral Baikal } \\
\hline 611028 & 53.60 & 108.80 & 4 & 42 & -124 & 5.5 & Irkutsk \\
\hline 620813 & 53.70 & 108.50 & 32 & 40 & -117 & 5.2 & Irkutsk \\
\hline 621028 & 53.60 & 108.60 & 8 & 44 & -120 & 3.5 & Irkutsk \\
\hline 630131 & 53.10 & 107.70 & 22 & 67 & -117 & 4.0 & Irkutsk \\
\hline 660403 & 54.00 & 108.60 & 37 & 33 & -122 & 4.5 & Irkutsk \\
\hline 720809 & 52.80 & 107.73 & 225 & 60 & -102 & 5.2 & Irkutsk \\
\hline
\end{tabular}


Table 1. (continued)

\begin{tabular}{|c|c|c|c|c|c|c|c|}
\hline Date / Information & Latitude & Longitude & Strike & Dip & Rake & Magnitude & Origin \\
\hline 820128 & 53.49 & 108.69 & 52 & 54 & -81 & 4.6 & Irkutsk \\
\hline 841209 & 53.83 & 108.59 & 17 & 30 & -117 & 4.8 & Irkutsk \\
\hline 871224 & 52.97 & 107.37 & 19 & 34 & -100 & 4.4 & Irkutsk \\
\hline 871224 & 52.96 & 107.40 & 335 & 42 & -126 & 4.5 & Irkutsk \\
\hline 900520 & 53.07 & 108.02 & 197 & 42 & -131 & 4.6 & Irkutsk \\
\hline 920214 & 53.88 & 108.87 & 248 & 33 & -66 & 5.4 & Irkutsk \\
\hline Composite & 52.85 & 107.04 & 321 & 32 & -65 & & Irkutsk \\
\hline Composite & 53.23 & 107.75 & 80 & 33 & -65 & & Irkutsk \\
\hline Composite & 53.15 & 107.72 & 22 & 67 & -117 & & Irkutsk \\
\hline Composite & 53.37 & 108.33 & 17 & 60 & -88 & & Irkutsk \\
\hline Composite & 53.38 & 108.34 & 53 & 56 & -105 & & Irkutsk \\
\hline Composite & 53.42 & 108.24 & 56 & 38 & -74 & & Irkutsk \\
\hline Composite & 53.62 & 108.19 & 3 & 46 & -98 & & Irkutsk \\
\hline Composite & 53.76 & 108.92 & 28 & 41 & -112 & & Irkutsk \\
\hline Composite & 52.85 & 107.04 & 321 & 32 & -65 & & Irkutsk \\
\hline \multicolumn{8}{|c|}{ Region: Barguzin } \\
\hline 610727 & 54.10 & 110.00 & 221 & 62 & -64 & 4.8 & Irkutsk \\
\hline 620111 & 54.50 & 111.00 & 204 & 40 & -101 & 2.8 & Irkutsk \\
\hline 630205 & 54.30 & 111.40 & 37 & 50 & -104 & 4.0 & Irkutsk \\
\hline 630214 & 54.80 & 111.90 & 228 & 50 & -105 & 3.3 & Irkutsk \\
\hline 630215 & 55.20 & 111.00 & 30 & 65 & -106 & 4.5 & Irkutsk \\
\hline 630411 & 54.40 & 111.40 & 38 & 50 & -112 & 3.5 & Irkutsk \\
\hline 770824 & 54.12 & 110.44 & 192 & 46 & -121 & 5.0 & Irkutsk \\
\hline 790110 & 55.43 & 111.44 & 190 & 51 & -116 & 5.0 & Irkutsk \\
\hline 790110 & 55.40 & 111.43 & 257 & 62 & -90 & 5.0 & Irkutsk \\
\hline 790211 & 55.42 & 111.44 & 205 & 60 & -109 & 3.5 & Irkutsk \\
\hline 791205 & 55.32 & 111.39 & 219 & 51 & -102 & 4.5 & Irkutsk \\
\hline 800404 & 54.67 & 109.80 & 21 & 30 & -90 & 4.5 & Irkutsk \\
\hline $810527^{*}$ & 54.03 & 109.42 & 251 & 67 & -87 & 5.2 & CMTS \\
\hline $820114^{*}$ & 54.76 & 110.28 & 206 & 80 & 174 & 4.9 & Doser \\
\hline 850223 & 55.37 & 111.33 & 22 & 52 & -74 & 4.0 & Irkutsk \\
\hline 850815 & 54.65 & 110.16 & 6 & 42 & -97 & 4.0 & Irkutsk \\
\hline 860122 & 55.46 & 109.37 & 10 & 50 & -114 & 4.6 & Irkutsk \\
\hline 870221 & 54.40 & 110.33 & 176 & 33 & -114 & 4.9 & CMTS \\
\hline 871008 & 55.29 & 109.60 & 112 & 40 & -42 & 4.0 & Irkutsk \\
\hline 890703 & 53.91 & 110.32 & 229 & 42 & -108 & 4.5 & Irkutsk \\
\hline 910912 & 54.68 & 110.70 & 28 & 67 & -101 & 5.2 & CMTS \\
\hline Composite & 53.88 & 109.02 & 172 & 42 & -113 & & Irkutsk \\
\hline Composite & 54.98 & 109.46 & 2 & 70 & -104 & & Irkutsk \\
\hline Composite & 55.33 & 109.62 & 28 & 46 & -76 & & Irkutsk \\
\hline Composite & 54.71 & 110.98 & 35 & 36 & -100 & & Irkutsk \\
\hline Composite & 54.73 & 111.04 & 12 & 58 & -117 & & Irkutsk \\
\hline Composite & 54.26 & 110.67 & 33 & 34 & -114 & & Irkutsk \\
\hline Composite & 53.77 & 110.21 & 29 & 42 & -129 & & Irkutsk \\
\hline Composite & 55.00 & 111.54 & 28 & 30 & -115 & & Irkutsk \\
\hline Composite & 54.79 & 111.93 & 36 & 32 & -101 & & Irkutsk \\
\hline Composite & 54.32 & 111.30 & 23 & 43 & -117 & & Irkutsk \\
\hline Composite & 54.32 & 111.30 & 39 & 49 & -113 & & Irkutsk \\
\hline Composite & 54.32 & 111.30 & 40 & 50 & -101 & & Irkutsk \\
\hline Composite & 54.31 & 111.39 & 51 & 85 & -98 & & Irkutsk \\
\hline Composite & 54.31 & 111.45 & 51 & 48 & -24 & & Irkutsk \\
\hline Composite & 55.17 & 111.04 & 33 & 50 & -110 & & Irkutsk \\
\hline Composite & 55.24 & 110.92 & 1 & 55 & -108 & & Irkutsk \\
\hline Composite & 55.18 & 110.19 & 310 & 55 & -100 & & Irkutsk \\
\hline Composite & 55.26 & 110.06 & 194 & 20 & -95 & & Irkutsk \\
\hline Composite & 55.15 & 110.19 & 19 & 86 & -44 & & Irkutsk \\
\hline Composite & 55.02 & 110.70 & 29 & 38 & -102 & & Irkutsk \\
\hline Composite & 55.25 & 111.17 & 16 & 49 & -112 & & Irkutsk \\
\hline Composite & 55.38 & 111.45 & 190 & 51 & -117 & & Irkutsk \\
\hline \multicolumn{8}{|c|}{ Region: Kulchera } \\
\hline 611123 & 55.85 & 110.15 & 78 & 50 & -90 & 4.5 & Irkutsk \\
\hline 661231 & 55.60 & 110.80 & 205 & 39 & -97 & 4.0 & Irkutsk \\
\hline
\end{tabular}


Table 1. (continued)

\begin{tabular}{|c|c|c|c|c|c|c|c|}
\hline Date / Information & Latitude & Longitude & Strike & Dip & Rake & Magnitude & Origin \\
\hline 670115 & 55.60 & 110.80 & 200 & 46 & -130 & 5.2 & Irkutsk \\
\hline 680617 & 55.96 & 110.58 & 69 & 56 & -136 & 4.5 & Irkutsk \\
\hline 720104 & 55.82 & 110.56 & 80 & 52 & -100 & 4.5 & Irkutsk \\
\hline 760923 & 55.75 & 110.54 & 37 & 68 & -140 & 5.0 & Irkutsk \\
\hline 821003 & 55.98 & 110.91 & 219 & 64 & -124 & 4.5 & Irkutsk \\
\hline $901026^{*}$ & 56.26 & 110.62 & 214 & 56 & -150 & 5.1 & CMTS \\
\hline Composite & 55.82 & 110.12 & 222 & 36 & -125 & & Irkutsk \\
\hline Composite & 55.81 & 110.24 & 71 & 54 & -97 & & Irkutsk \\
\hline Composite & 55.90 & 110.96 & 75 & 78 & -93 & & Irkutsk \\
\hline Composite & 55.82 & 110.66 & 76 & 58 & -101 & & Irkutsk \\
\hline Composite & 55.72 & 110.59 & 37 & 68 & -141 & & Irkutsk \\
\hline Composite & 55.73 & 110.57 & 17 & 54 & -123 & & Irkutsk \\
\hline Composite & 55.61 & 110.71 & 215 & 60 & -104 & & Irkutsk \\
\hline \multicolumn{8}{|c|}{ Region: Tsipa } \\
\hline 680721 & 55.18 & 113.45 & 71 & 70 & -95 & 5.0 & Irkutsk \\
\hline 730616 & 54.85 & 112.58 & 194 & 44 & -122 & 5.1 & Irkutsk \\
\hline 860520 & 55.25 & 113.35 & 278 & 64 & -123 & 4.0 & Irkutsk \\
\hline 900718 & 54.96 & 112.23 & 206 & 47 & -111 & 4.5 & Irkutsk \\
\hline Composite & 54.87 & 112.50 & 43 & 50 & -87 & & Irkutsk \\
\hline Composite & 55.24 & 113.21 & 53 & 30 & -109 & & Irkutsk \\
\hline Composite & 55.25 & 113.33 & 74 & 73 & -94 & & Irkutsk \\
\hline Composite & 55.38 & 113.58 & 26 & 52 & -102 & & Irkutsk \\
\hline \multicolumn{8}{|c|}{ Region: Upper Angara } \\
\hline 630115 & 55.80 & 112.90 & 210 & 65 & -127 & 3.5 & Irkutsk \\
\hline 630312 & 56.10 & 111.50 & 57 & 61 & -104 & 3.5 & Irkutsk \\
\hline 630318 & 56.00 & 112.20 & 92 & 36 & -90 & 3.5 & Irkutsk \\
\hline 631201 & 55.90 & 112.00 & 28 & 26 & -106 & 4.9 & Irkutsk \\
\hline 671016 & 55.90 & 111.10 & 68 & 58 & -112 & 4.0 & Irkutsk \\
\hline 681126 & 55.90 & 111.49 & 264 & 55 & -91 & 5.3 & Irkutsk \\
\hline 761102 & 56.19 & 111.59 & 32 & 50 & -123 & 5.2 & Irkutsk \\
\hline 770604 & 56.20 & 111.82 & 22 & 22 & -132 & 4.7 & Irkutsk \\
\hline 790221 & 55.84 & 111.31 & 92 & 53 & -98 & 4.0 & Irkutsk \\
\hline 790701 & 55.66 & 112.30 & 259 & 74 & -128 & 4.0 & Irkutsk \\
\hline 810225 & 56.22 & 111.57 & 46 & 54 & -56 & 4.5 & Irkutsk \\
\hline 810303 & 55.73 & 112.88 & 65 & 50 & -98 & 4.2 & Irkutsk \\
\hline 810531 & 56.17 & 111.76 & 189 & 50 & -120 & 4.5 & Irkutsk \\
\hline 851111 & 55.63 & 112.01 & 327 & 54 & -118 & 4.2 & Irkutsk \\
\hline 881216 & 56.08 & 111.66 & 68 & 54 & -20 & 4.0 & Irkutsk \\
\hline Composite & 55.98 & 111.53 & 264 & 55 & -91 & & Irkutsk \\
\hline Composite & 55.96 & 111.25 & 73 & 58 & -99 & & Irkutsk \\
\hline Composite & 55.51 & 111.43 & 31 & 43 & -109 & & Irkutsk \\
\hline Composite & 55.60 & 111.96 & 205 & 46 & -94 & & Irkutsk \\
\hline Composite & 56.15 & 112.30 & 56 & 50 & -111 & & Irkutsk \\
\hline Composite & 56.36 & 112.55 & 33 & 41 & -125 & & Irkutsk \\
\hline Composite & 56.23 & 112.44 & 198 & 21 & -116 & & Irkutsk \\
\hline Composite & 56.24 & 112.69 & 26 & 44 & -133 & & Irkutsk \\
\hline Composite & 55.81 & 113.00 & 50 & 48 & -111 & & Irkutsk \\
\hline \multicolumn{8}{|c|}{ Region: Muyakan } \\
\hline 620810 & 56.50 & 113.80 & 221 & 50 & -101 & 3.3 & Irkutsk \\
\hline 621111 & 55.84 & 113.22 & 215 & 58 & -78 & 5.7 & Doser \\
\hline 681108 & 56.14 & 113.75 & 204 & 47 & -107 & 4.5 & Irkutsk \\
\hline 740701 & 56.09 & 113.81 & 262 & 80 & 88 & 5.0 & Irkutsk \\
\hline 781021 & 56.31 & 113.24 & 17 & 67 & 66 & 2.8 & Irkutsk \\
\hline 790415 & 56.33 & 113.42 & 269 & 70 & -121 & 4.0 & Irkutsk \\
\hline 840202 & 55.93 & 113.68 & 96 & 44 & -100 & 4.0 & Irkutsk \\
\hline 880604 & 55.87 & 113.18 & 54 & 35 & 35 & 4.3 & Irkutsk \\
\hline Composite & 56.38 & 113.33 & 270 & 15 & -94 & & Irkutsk \\
\hline Composite & 56.34 & 113.31 & 61 & 46 & -111 & & Irkutsk \\
\hline Composite & 56.33 & 113.34 & 299 & 39 & -134 & & Irkutsk \\
\hline Composite & 56.31 & 113.51 & 267 & 88 & -19 & & Irkutsk \\
\hline Composite & 56.16 & 113.45 & 86 & 62 & -92 & & Irkutsk \\
\hline Composite & 56.32 & 113.60 & 80 & 76 & -93 & & Irkutsk \\
\hline
\end{tabular}


Table 1. (continued)

\begin{tabular}{|c|c|c|c|c|c|c|c|}
\hline Date / Information & Latitude & Longitude & Strike & Dip & Rake & Magnitude & Origin \\
\hline Composite & 56.30 & 113.15 & 234 & 78 & -104 & & Irkutsk \\
\hline Composite & 56.14 & 113.19 & 35 & 66 & 73 & & Irkutsk \\
\hline Composite & 56.33 & 113.51 & 253 & 44 & -115 & & Irkutsk \\
\hline Composite & 56.27 & 113.49 & 4 & 80 & -74 & & Irkutsk \\
\hline Composite & 56.31 & 113.51 & 255 & 52 & -110 & & Irkutsk \\
\hline Composite & 56.29 & 113.42 & 280 & 66 & -116 & & Irkutsk \\
\hline Composite & 56.34 & 113.46 & 258 & 39 & -155 & & Irkutsk \\
\hline Composite & 56.31 & 113.53 & 342 & 8 & 81 & & Irkutsk \\
\hline Composite & 56.20 & 113.61 & 220 & 83 & -30 & & Irkutsk \\
\hline Composite & 56.20 & 113.39 & 254 & 84 & -94 & & Irkutsk \\
\hline Composite & 56.21 & 113.51 & 225 & 50 & 180 & & Irkutsk \\
\hline Composite & 56.39 & 113.83 & 70 & 58 & -103 & & Irkutsk \\
\hline Composite & 56.39 & 113.81 & 225 & 30 & -22 & & Irkutsk \\
\hline Composite & 56.33 & 113.87 & 247 & 36 & -96 & & Irkutsk \\
\hline Composite & 56.20 & 113.91 & 70 & 66 & -100 & & Irkutsk \\
\hline Composite & 56.19 & 113.84 & 69 & 76 & -90 & & Irkutsk \\
\hline Composite & 56.21 & 113.93 & 15 & 27 & -146 & & Irkutsk \\
\hline Composite & 56.09 & 113.91 & 220 & 17 & -106 & & Irkutsk \\
\hline Composite & 56.04 & 113.70 & 78 & 29 & -113 & & Irkutsk \\
\hline Composite & 56.05 & 113.73 & 69 & 62 & -117 & & Irkutsk \\
\hline Composite & 56.04 & 113.73 & 267 & 13 & -160 & & Irkutsk \\
\hline Composite & 56.02 & 113.73 & 229 & 33 & -113 & & Irkutsk \\
\hline Composite & 55.86 & 113.26 & 87 & 85 & -96 & & Irkutsk \\
\hline Composite & 55.98 & 113.69 & 48 & 50 & -96 & & Irkutsk \\
\hline Composite & 55.79 & 113.46 & 54 & 53 & -90 & & Irkutsk \\
\hline Composite & 55.95 & 113.93 & 65 & 66 & -118 & & Irkutsk \\
\hline Composite & 56.00 & 113.41 & 79 & 31 & -104 & & Irkutsk \\
\hline Composite & 55.81 & 113.00 & 50 & 48 & -111 & & Irkutsk \\
\hline Composite & 55.82 & 114.00 & 56 & 63 & -101 & & Irkutsk \\
\hline \multicolumn{8}{|c|}{ Region: Muya } \\
\hline $570627^{*}$ & 56.20 & 116.54 & 120 & 80 & -40 & 7.8 & Doser \\
\hline 680831 & 56.40 & 115.78 & 245 & 60 & -107 & 5.0 & Irkutsk \\
\hline 711218 & 56.19 & 114.21 & 78 & 84 & -97 & 5.0 & Irkutsk \\
\hline 771120 & 56.55 & 115.78 & 71 & 55 & -99 & 4.0 & Irkutsk \\
\hline 860517 & 56.06 & 114.84 & 351 & 64 & -142 & 3.5 & Irkutsk \\
\hline 860526 & 56.26 & 116.19 & 120 & 46 & 83 & 4.2 & Irkutsk \\
\hline 880621 & 56.07 & 114.68 & 347 & 29 & -127 & 4.0 & Irkutsk \\
\hline Composite & 56.09 & 114.02 & 34 & 65 & -96 & & Irkutsk \\
\hline Composite & 56.23 & 114.14 & 78 & 84 & -97 & & Irkutsk \\
\hline Composite & 56.41 & 114.28 & 268 & 15 & -112 & & Irkutsk \\
\hline Composite & 56.34 & 114.42 & 49 & 72 & -106 & & Irkutsk \\
\hline Composite & 56.12 & 114.51 & 30 & 41 & -108 & & Irkutsk \\
\hline Composite & 56.18 & 114.85 & 31 & 51 & -113 & & Irkutsk \\
\hline Composite & 56.18 & 114.87 & 68 & 72 & -95 & & Irkutsk \\
\hline Composite & 56.32 & 115.51 & 45 & 26 & -112 & & Irkutsk \\
\hline Composite & 56.29 & 115.50 & 248 & 28 & -99 & & Irkutsk \\
\hline Composite & 56.25 & 116.51 & 68 & 55 & -91 & & Irkutsk \\
\hline Composite & 56.20 & 116.76 & 322 & 56 & -109 & & Irkutsk \\
\hline Composite & 56.28 & 116.39 & 6 & 44 & 135 & & Irkutsk \\
\hline Composite & 56.21 & 116.37 & 59 & 39 & -91 & & Irkutsk \\
\hline \multicolumn{8}{|c|}{ Region: Tchara } \\
\hline 620423 & 56.50 & 117.20 & 210 & 52 & -119 & 3.3 & Irkutsk \\
\hline 700515 & 56.84 & 117.74 & 193 & 72 & 176 & 5.5 & Irkutsk \\
\hline 700518 & 56.87 & 117.87 & 76 & 10 & -90 & 4.8 & Irkutsk \\
\hline 740621 & 56.35 & 117.70 & 40 & 58 & -133 & 5.1 & Irkutsk \\
\hline 750206 & 56.41 & 117.89 & 236 & 79 & -51 & 4.7 & Irkutsk \\
\hline 810117 & 56.36 & 117.94 & 21 & 45 & -141 & 5.1 & Irkutsk \\
\hline 840619 & 56.45 & 118.25 & 66 & 56 & -64 & 4.7 & Irkutsk \\
\hline 891205 & 56.67 & 117.99 & 186 & 42 & -69 & 4.5 & Irkutsk \\
\hline 940821 & 56.57 & 117.85 & 43 & 47 & -104 & 6.0 & CMTS \\
\hline Composite & 56.48 & 117.17 & 86 & 64 & 2 & & Irkutsk \\
\hline Composite & 56.48 & 117.19 & 129 & 62 & -100 & & Irkutsk \\
\hline
\end{tabular}


Table 1. (continued)

\begin{tabular}{llllllll}
\hline Date / Information & Latitude & Longitude & Strike & Dip & Rake & Magnitude & Origin \\
\hline Composite & 56.69 & 117.33 & 229 & 43 & -107 & & Irkutsk \\
Composite & 56.67 & 117.37 & 32 & 34 & -148 & & Irkutsk \\
Composite & 56.60 & 117.74 & 50 & 35 & -101 & & Irkutsk \\
Composite & 56.60 & 117.78 & 311 & 44 & 74 & & Irkutsk \\
Composite & 56.62 & 117.73 & 256 & 54 & -107 & & Irkutsk \\
Composite & 56.37 & 117.47 & 70 & 69 & -116 & & Irkutsk \\
Composite & 56.27 & 117.72 & 31 & 64 & -109 & & Irkutsk \\
Composite & 56.41 & 117.58 & 40 & 58 & -131 & & Irkutsk \\
Composite & 56.39 & 118.12 & 28 & 62 & -113 & & Irkutsk \\
Composite & 56.40 & 118.18 & 24 & 48 & -60 & & Irkutsk \\
Composite & 56.68 & 118.28 & 68 & 80 & -35 & & Irkutsk \\
Composite & 56.18 & 117.30 & 83 & 47 & -117 & & Irkutsk \\
& & & Region: Eastern rift & & \\
$580105^{*}$ & 56.51 & 121.11 & 257 & 50 & -100 & 6.5 & Doser \\
$580914^{*}$ & 56.61 & 121.06 & 63 & 63 & -75 & 6.3 & Doser \\
$670118^{*}$ & 56.54 & 120.93 & 59 & 66 & -166 & 6.0 & Doser \\
710617 & 56.10 & 123.60 & 264 & 73 & -8 & 5.7 & Doser \\
720115 & 57.50 & 121.10 & 102 & 62 & -61 & 4.8 & Irkutsk \\
721125 & 56.19 & 123.56 & 45 & 74 & -169 & 5.0 & Doser \\
$870707^{*}$ & 56.67 & 121.59 & 260 & 27 & -90 & 5.3 & CMTS \\
890420 & 57.03 & 121.23 & 101 & 82 & 33 & 6.3 & CMTS \\
890429 & 57.13 & 121.80 & 91 & 87 & -17 & 5.4 & CMTS \\
890507 & 57.04 & 122.28 & 3 & 76 & 18 & 4.5 & Doser \\
890517 & 57.07 & 122.03 & 277 & 77 & -43 & 6.0 & CMTS \\
\hline
\end{tabular}

See Figure 2 for geographical positions of the subregions. "Irkutsk" and "Novosibirsk" origins correspond to Russian publications of focal mechanisms from both Institutes [see e.g., Solonenko et al., 1993]. "Doser" origin refers to Doser [1991a,b] modelings. Asterisks indicate fault plane solutions determined by several procedures (waveform modeling and first-motion determinations).

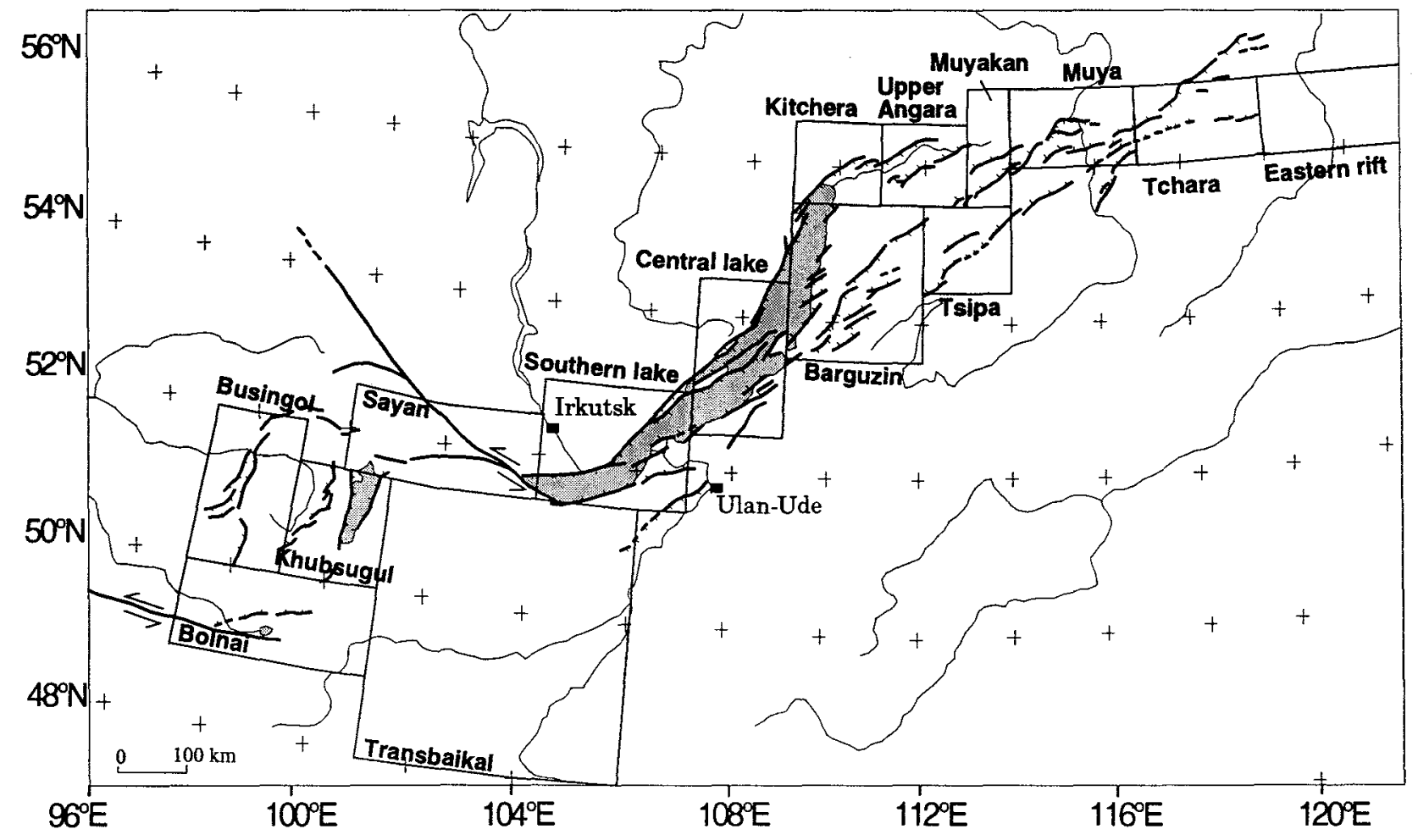

Figure 1. General map of the Baikal rift zone showing main active faults (bold lines) and 15 subareas (rectangles) corresponding to stress tensor determinations. 


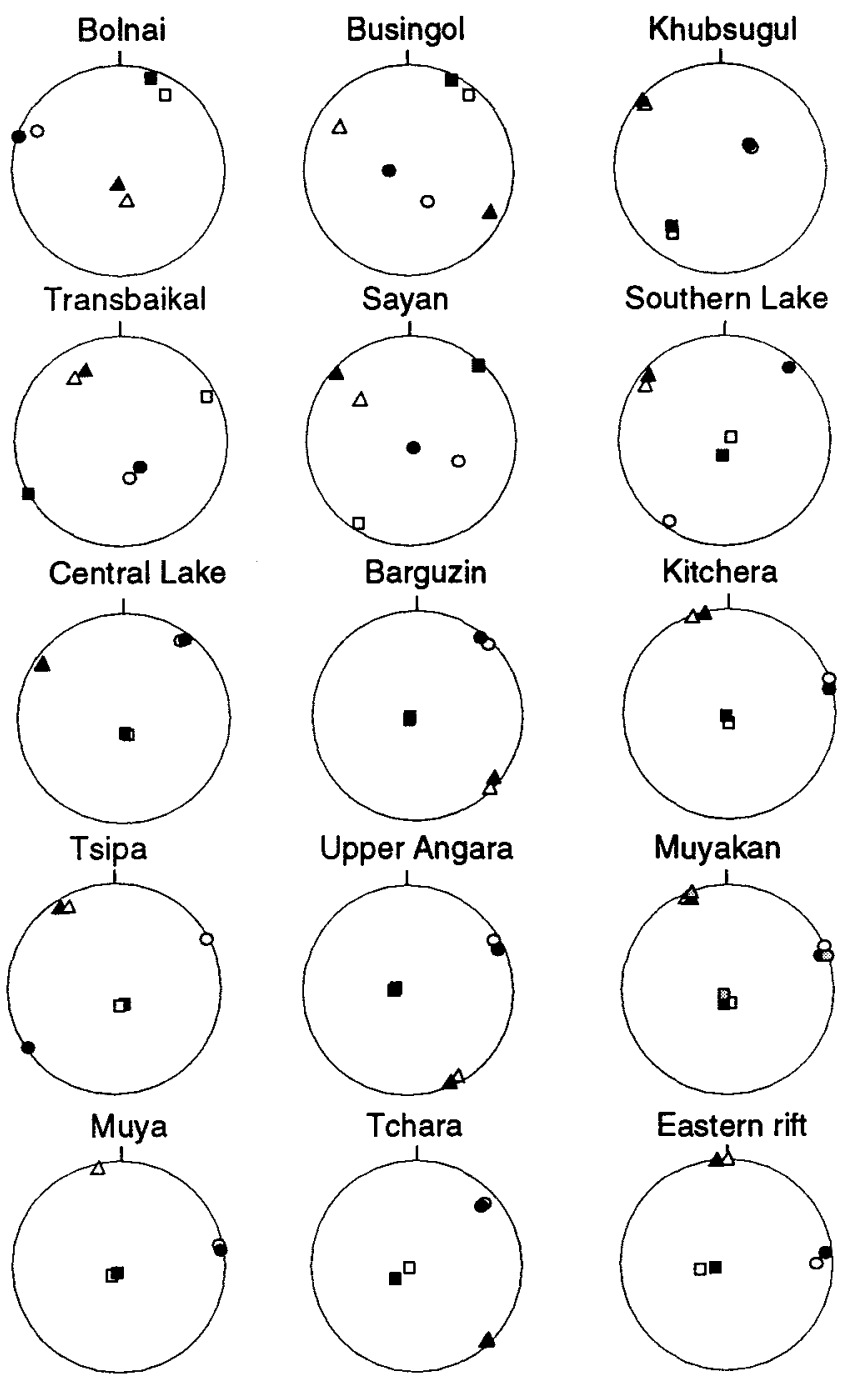

Figure 2. Main stress directions determined by numerical methods of Carey and Mercier [1987] (open symbols) and Delvaux et al. [1996] (solid symbols). Grey symbols for the Muyakan area are results after Déverchère et al. [1993]. Squares, circles and triangles are $\sigma_{1}, \sigma_{2}$, and $\sigma_{3}$ axes, respectively.

\section{Predicted Slip Vectors}

A stress tensor can be used to predict the direction and sense of movement on a given fault plane, especially in cases where field data are scarce or not reliable. Ritz [1994] developed a simple graphical method allowing one to deduce the slip vector on a given fault plane from the stress ellipsoid. We apply this method to several well-recognized faults in the BRZ, using the corresponding stress tensors previously obtained, in order to determine the predicted movement associated with the local stress field (Table 4). Compared to the use of focal mechanisms, this allows us to avoid possible large errors linked to uncertainties on positions of nodal poles: the stress tensor is well constrained by a large enough amount of data, and thus considerably reduces the uncertainty linked to the use of individual earthquakes. Moreover, active fault geometry is integrated during this procedure, which is supposed to provide more realistic estimates of slip vectors.

\section{Active Fault Patterns}

Detailed morphological and field analyses have been made in the NBR by Houdry [1994]. In this part of the rift, major fault scarps are numerous and shorter than in the SBR. They have been identified and measured either on the field or on satellite (SPOT) images. We gather the information on main fault direction and plunge in the NBR from this source (Table 4), considering the border faults of Kitchera, Upper Angara, Barguzin, Tsipa, Upper Muya, Muyakan, Muya, and Tchara basins as main active faults (Figure 5). In the NBR, faults exhibit high dips ranging between $50^{\circ}$ and $60^{\circ}$ [Déverchère et al., 1993; Houdry, 1994]. Fault scarps strike from NS (locally in the Barguzin basin) to EW (in the Muya basin), but most of them strike approximately $N 60^{\circ} \mathrm{E}$. This direction is inherited from anterift Paleozoic and Cenozoic history [Sherman, 1978; Ermikov, 1994; Delvaux et al., 1996].

The Sayan-Tunka region is the southern limit of the studied area, because accuracies on fault geometry and stress tensors are not good enough to deduce slip vectors further southward. Deformation in the SBR is highly localized and mainly implies three major active faults: the South Baikal, Sayan, and Tunka faults. Information on their geometry comes from satellite (SPOT) images and Russian field studies [Sherman, 1992]. The South Baikal and Tunka faults strike about $\mathrm{N} 80^{\circ} \mathrm{E}$ and dip southwards. The vertical dip of the Sayan fault is well evidenced by a nearly linear track, quite visible for $50 \mathrm{~km}$ from the southern tip of Baikal lake toward the NW.

In order to predict slip vectors on these structures, we make the assumption that the stress field determined from fault plane solutions is responsible for the movement along major faults. Indeed, seismicity distribution suggests that active deformation is preferably concentrated on these faults which correspond to inherited directions, except swarms that occur at the junction between the basins and may involve incipient neoforming structures [Solonenko, 1985; Petit, 1993]. Table 4 shows the resulting slip vectors predicted on these faults and the corresponding sense of movement. In order to estimate uncertainties on slip vector azimuths, we have computed the errors on predicted striations resulting from slight changes (equal to estimated uncertainties) applied to the stress tensor.

\section{Slip Distribution}

Figure 6 shows directions of horizontal movement predicted on main faults. Their azimuths vary from $\mathrm{N} 73^{\circ} \mathrm{E}$ to $\mathrm{N} 177^{\circ} \mathrm{W}$, lineating different "kinematic provinces". Most of the predicted slip vectors (Table 4 and Figure 6) depict normal faulting, with a variable amount of strike-slip (e.g., sinistral for South Angara 2, Tchara, Barguzin 3, Primorsky 1 and Southern lake faults; dextral for Muyakan, Barguzin 1, and Taksimo faults). Sinistral strikeslip faulting with a small reverse component is found along the Sayan fault. In the SBR, including the southern half of the lake, slip directions are rather stable and range between $\mathrm{N} 107^{\circ} \mathrm{E}$ and $\mathrm{N} 121^{\circ} \mathrm{E}$, except for the Tunka fault which exhibits a $\mathrm{N} 73^{\circ} \mathrm{E}$ trending slip vector. This southern area of narrow and localized rifting is thus characterized by a relatively simple fault system exhibiting important sinistral movement. Note that this part of the rift is located close to the Paleozoic suture separating the Siberian craton from the Sayan-Baikal folded zone (Figure 5). The position of the rift at the emplacement of this major structural discontinuity [Zamarayev and Ruzhich, 1978; Logatchev, 1993] 


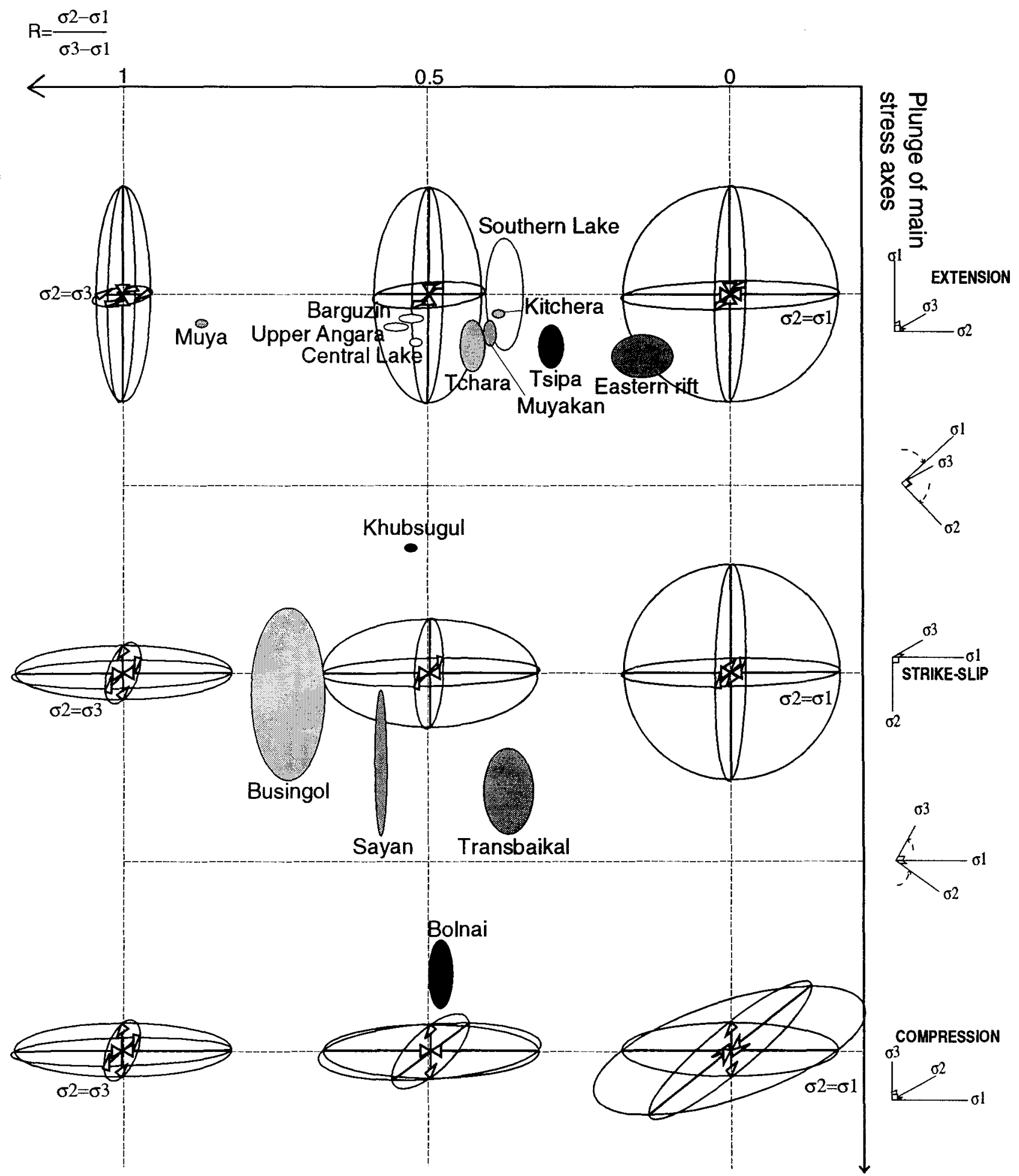

Figure 3. Diagram showing stress regime changes from radial extensive (upper left ellipsoid) to radial compressive (lower-right ellipsoid) cases. $R$ ratio varies horizontally. Inclination of main stress axes varies vertically. Ellipses correspond to uncertainty estimations on stress tensors. Grey levels refer to the quality factors: white, light grey, dark grey and black correspond to A, B, C, and D factors, respectively (see Table 2).

may explain the localized character of the deforming zone, as well as the homogeneous motion direction.

North of $54^{\circ} \mathrm{N}$, slip distribution is much more variable (between $\mathrm{N} 116^{\circ} \mathrm{E}$ and $\mathrm{N} 177^{\circ} \mathrm{W}$ ). Several features are standing out in Figure 6: along the southern boundary of the deformed zone, predicted motions along the Barguzin and Tsipa faults are rather stable (around $\mathrm{N} 145^{\circ} \mathrm{E}$ ); meanwhile, the northern boundary exhibits more complex features which are characterized by 


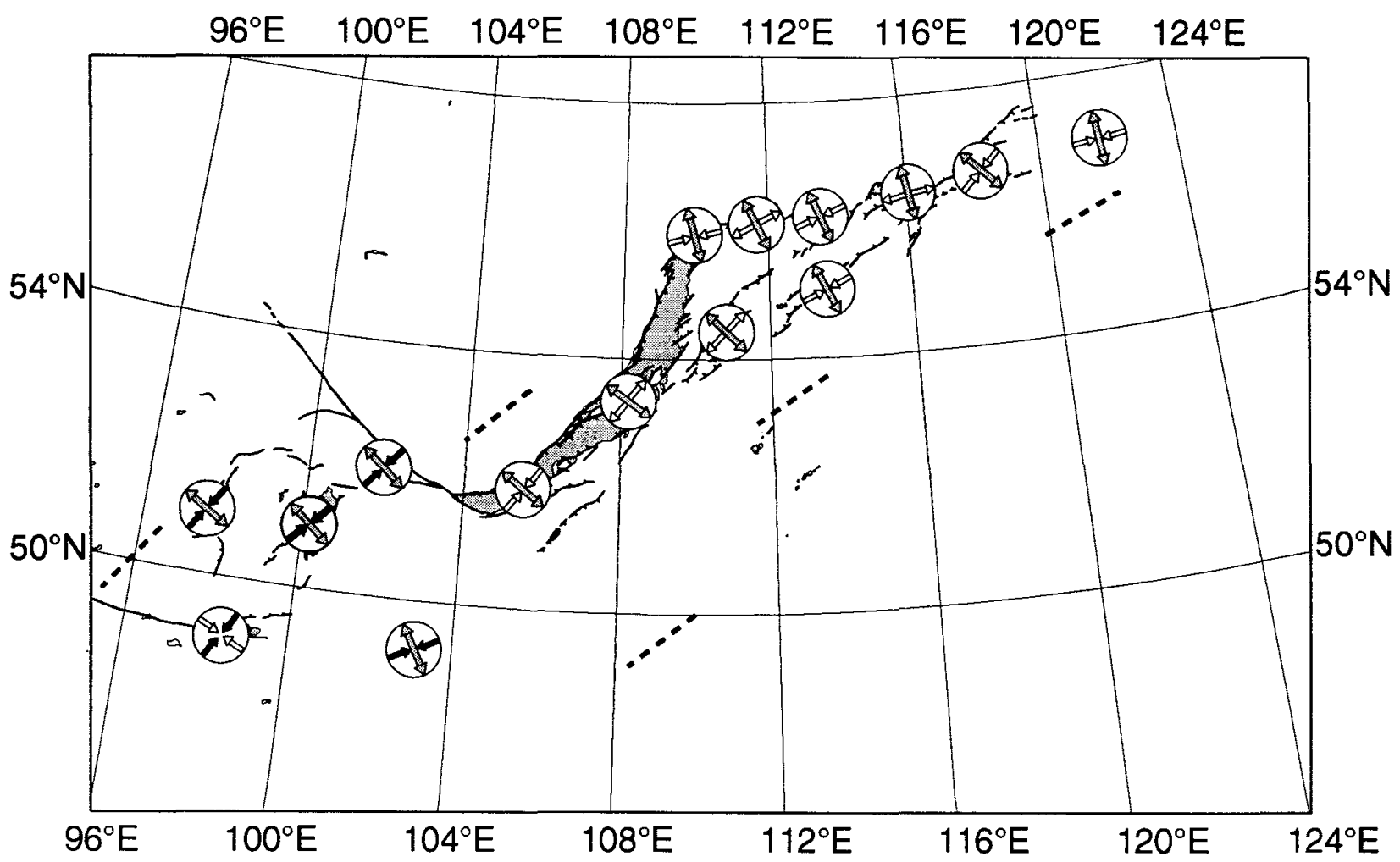

Figure 4. Map view of main horizontal stress directions in the Baikal rift (see Table 2). Grey arrows are $\sigma_{3}$; open divergent arrows are $\sigma_{2}$ when $R>0.5$; open convergent arrows are $\sigma_{2}$ when $R<0.5$; solid arrows are $\sigma_{1}$. Thick dashed line is regional $S_{H \max }$ direction after Tapponnier and Molnar [1979] and Zoback [1992].

progressive counterclockwise rotations of slip vectors from about $\mathrm{N} 180^{\circ} \mathrm{E}$ to $\mathrm{N} 130^{\circ} \mathrm{E}$. From west to east, these rotations appear twice and indicate two main systems: the upper Angara (UA) area and the Muyakan-Upper Muya (M-UM) area (Figure 6). What is the meaning of such a complex movement pattern? Seismicity distribution in the NBR shows a pattern of seismic belts and swarms, clearly delimiting two aseismic zones. The first one includes the UA and M-UM areas, and the second one seems to correspond to the Barguzin-Tsipa (BT) region (Figure 7). Moreover, the boundary between UA and M-UM blocks is well underlined by a dextral shift of the corresponding seismic belts. From these observations, we infer that the deformation along the northern boundary of the NBR is characterized by block rotations, involving two crustal blocks rotating counterclockwise inside the deformed zone, relatively to the northern edge of the rift. The mean slip vector between the third block (BT) and the southem rift boundary has a constant azimuth.

\section{Consistency With Previous Studies}

These predicted motions along major rift faults are generally in good agreement with previous field observations and computed motions from cumulated Holocene displacements [see e.g., Houdry, 1994], and with previous local stress tensor determination [Déverchère et al., 1993]. These studies (including this one) tend to indicate a dominant component of normal faulting in the NBR, which invalidates the strong sinistral component predicted by several authors [Balla et al., 1991;
Ruppel et al., 1993] along the NBR faults. Oblique opening on the South Baikal fault and sinistral strike-slip motion on the Sayan fault are also consistent with previous results [see e.g., Zonenshain and Savostin, 1980; Sherman, 1978]. However, slip vector prediction shows two discrepancies with field analysis results. First, the computed fault motion on the Kitchera fault depicts a normal-dextral motion (Figure 6), while morphological field studies evidence normal-sinistral displacement [Houdry, 1994]. In order to explain this discrepancy, we assume that the Kitchera fault may not really belong to the UA block, and should be instead associated with the North Baikal basin system. The lack of seismicity in this latter area does not allow us to compute a stress tensor, but observed displacements along the North Baikal and Kitchera faults favor an average NW-SE trending slip. Second, the Tunka fault predicted slip vector depicts a reversesinistral motion; evidence for such movement is observed on the western part of the fault, but clear normal faulting is seen in its eastern half [see e.g., McCalpin and Khromovskikh, 1995]. The realistic sinistral strike-slip motion predicted for the Sayan fault leads us to believe that the stress tensor is reliable. The uncompatibility between predicted and observed motions on the eastern Tunka fault remains thus to be explained. It may result from a rapid change of the stress field in this region: the stress tensor computed on the western parts of Sayan and Tunka regions may be not suitable for slip vector computation in the eastern Tunka basin. A strain partitioning between the Sayan and Tunka faults may also be invoked. 
Table 4. Predicted Slip Vectors on Main Active Faults of the BRZ

\begin{tabular}{|c|c|c|c|c|}
\hline Name of the fault & Stress Tensor & Az. Pl. & $\begin{array}{l}\text { Predicted } \\
\text { Striation }\end{array}$ & $\begin{array}{c}\text { Esti- } \\
\text { mated } \\
1 \sigma\end{array}$ \\
\hline Tunka & Sayan & $\mathrm{N} 80,60 \mathrm{~S}$ & $253-12$ & 3 \\
\hline Sayan & Sayan & $\mathrm{N} 120,90$ & $301-27$ & 9 \\
\hline Southern lake 1 & Southern lake & $\mathrm{N} 75,60 \mathrm{~S}$ & $107-42$ & 11 \\
\hline Southern lake 2 & Southern lake & $\mathrm{N} 65,60 \mathrm{~N}$ & $285-48$ & 5 \\
\hline Primorsky 1 & Central lake & $\mathrm{N} 50,60 \mathrm{~S}$ & $110-56$ & 3 \\
\hline Primorsky 2 & Central lake & $\mathrm{N} 35,60 \mathrm{~S}$ & $135-60$ & 5 \\
\hline Svyatoy Nos & Central lake & $\mathrm{N} 30,60 \mathrm{~N}$ & $296-60$ & 3 \\
\hline Barguzin 1 & Barguzin & $\mathrm{N} 25,60 \mathrm{E}$ & $145-56$ & 6 \\
\hline Barguzin 2 & Barguzin & $\mathrm{N} 40,60 \mathrm{~S}$ & $139-60$ & 7 \\
\hline Barguzin 3 & Barguzin & $\mathrm{N} 65,60 \mathrm{~N}$ & $297-58$ & 5 \\
\hline Kitchera & Kitchera & $\mathrm{N} 60,60 \mathrm{~S}$ & $183-55$ & 11 \\
\hline Dzelinda & Kitchera & $\mathrm{N} 60,50 \mathrm{~S}$ & $172-48$ & 10 \\
\hline Tsipa 1 & Tsipa & $\mathrm{N} 60,60 \mathrm{~N}$ & $330-60$ & 15 \\
\hline Tsipa 2 & Tsipa & $\mathrm{N} 65,60 \mathrm{~N}$ & $325-60$ & 15 \\
\hline North Angara 1 & Upper Angara & $\mathrm{N} 60,60 \mathrm{~S}$ & $163-59$ & 4 \\
\hline North Angara 2 & Upper Angara & $\mathrm{N} 80,70 \mathrm{~S}$ & $134-66$ & 3 \\
\hline South Angara 1 & Upper Angara & N50,60N & $340-58$ & 7 \\
\hline South Angara 2 & Upper Angara & $N 75,60 \mathrm{~N}$ & $314-56$ & 6 \\
\hline Upper Muya 1 & Muyakan & $\mathrm{N} 60,60 \mathrm{~N}$ & $340-60$ & 11 \\
\hline Upper Muya 2 & Muyakan & $\mathrm{N} 55,60 \mathrm{~N}$ & $343-59$ & 12 \\
\hline Upper Muya 3 & Muya & $\mathrm{N} 50,60 \mathrm{~N}$ & $319-60$ & 11 \\
\hline Muyakan 1 & Muyakan & $\mathrm{N} 50,60 \mathrm{~N}$ & $345-59$ & 13 \\
\hline Muyakan 2 & Muyakan & $\mathrm{N} 75,60 \mathrm{~N}$ & $329-59$ & 7 \\
\hline Muya 1 & Muya & $\mathrm{N} 90,60 \mathrm{~S}$ & $181-60$ & 11 \\
\hline Muya 2 & Muya & $\mathrm{N} 40,60 \mathrm{~N}$ & $327-60$ & 12 \\
\hline Taksimo & Muya & $\mathrm{N} 40,60 \mathrm{~S}$ & $159-57$ & 9 \\
\hline Tchara & Tchara & N65,60S & $131-58$ & 18 \\
\hline
\end{tabular}

See Figure 5 for faull locations. Column 3 shows azimuth and plunge (in degrees) of the faults. Column 4 shows direction and plunge (in degrees) of predicted striations. Estimated $1 \sigma$ (column 5 ) is the computed error on each slip vector determination (see Table 2), which underestimates the actual error, according to the quality factor assigned to each stress tensor determination (see Table 2). BRZ is Baikal Rift Zone.

\section{Discussion: Comparison with the Asian Stress Field and Kinematics}

The stress field study and inferred fault motions in the BRZ have allowed us to draw the main characteristics of rift dynamics and kinematics. How far does the regional (Asian) tectonic regime affect the rift characteristics, such as north-south contrast, and block rotations in the NBR? Previous studies have shown that global stress field patterns in central Asia are characterized by a continuous variation both in N-S and E-W directions [Tapponnier and Molnar, 1979; Zoback, 1992]: from west to east, $S_{H \max }$ orientation varies from NW-SE in the Pamir-Tarim region to N-S in the Gobi-Altai and NE-SW in the Tibet, Tsaidam, Nan-Shan, and Baikal zones; from south to north, the main outstanding feature is the progressive evolution of the stress field from compressional (Tarim, Tibet, Tien Shan) to wrench (Bolnai, Gobi-Altai) and finally extensional in the Baikal zone.

This study allows us to compare the local stress field variations in the Baikal rift zone to the broad-scale extensional stress regime previously described in this region. A first observation is that the average local $S_{H \max }$ directions are generally consistent with regional ones, suggesting that the same cause (the India-Asia collision) is responsible both for wrenchcompressional tectonics existing in Mongolia and for the extensional regime governing the Baikal rift. However, some discrepancies are worth noting: in the SBR, while $S_{H \max }$ keeps striking NE-SW, the stress field abruptly changes from wrenchcompressional to extensional; in the NBR, strong local $S_{H \max }$ deviations are found. We infer that inherited structures have an important influence on these local stress field changes: as reported before, the rapid change of tectonic regime happening in the SBR is geographically located at the place where the craton boundary direction changes (Figure 5); moreover, stress deviations in the whole rift are not randomly occurring but seem strongly linked to active fault tectonics at the same scale. In the extensional regime (Central lake, Upper Angara, Barguzin and Muya), systematic deviations of $S_{\text {Hmax }}$ tend to trend parallel to normal fault directions. In the wrench-extensional regime (Southern lake, Kitchera, Tchara), the angle between $S_{H \max }$ and fault directions ranges between $13^{\circ}$ and $38^{\circ}$. Finally, in the wrench-compressional regime (Bolnai, $S$ ayan), $S_{\text {Hmax }}$ becomes nearly perpendicular $\left(65^{\circ}-80^{\circ}\right)$ to main fault orientations (Figure 8). Such stress reorientations close to major faults are also reported in the Mediterranean region by Rebaï et al. [1992]. Although broad-scale features of $S_{\text {Hmax }}$ directions are relatively stable across the Asian continent, local influences are thus needed to explain the local stress field of the Baikal rift. This observation does not allow us to disregard the possible effect of deep lithospheric perturbations as the cause of second-order stress patterns. However, it highlights a strong relationship of stress field variations to the varying geometry of active faults and ancient sutures, suggesting that preexisting structures at least partly control the state of stress of the rift.

Such interaction between local stress patterns and local structures finds its expression in the deformation of the rift zone, allowing us to draw a similar comparison with the global strain of Asia. The latter is still a subject of controversy; especially, the role of strike-slip faulting in the accommodation of India-Asia convergence is highly debated. According to several authors, extrusion processes involving localized deformation along major strike-slip faults are dominant [see e.g., Tapponnier et al., 1982; Peltzer and Tapponnier, 1988; Avouac and Tapponnier, 1993]. On the other hand, some authors have modelled a more continuous style of deformation, where strike-slip faulting plays a limited role in the accommodation of plate motion [see e.g., Houseman and England, 1993]. Recently, from modelling of earthquake moment tensors, Holt et al. [1995] have proposed that strike-slip faulting only accommodates the rotation of the South China block relative to Siberia. Generally, although several authors agree that the India-Asia collision effects extend as far as in the Baikal region [see e.g., Molnar and Tapponnier, 1975; Molnar and Deng, 1984], only few models predict consequent horizontal strains in the BRZ. Molnar and Deng [1984] use large earthquakes to predict an average convergence azimuth of $\mathrm{N} 36^{\circ} \mathrm{E}$ between Mongolia and Siberia. Holt et al. [1995] also modelled an average SW-NE motion of Mongolia relative to Siberia, south of the Baikal Rift Zone. In spite of large uncertainties, both models favor SW-NE convergence between Mongolia (Amurian plate) and Siberia, which is quite consistent with the maximum horizontal stress direction observed.

At a more detailed scale, the BRZ depicts abrupt lateral strain variations. From south to north, reverse, oblique, and normal motions follow each other. It thus remains to explain how far the rift deformation described here fits or does not fit the kinematic model of central Asia, that is, what are the causes of opening of the rift in a context of SW-NE plate convergence? From a purely 


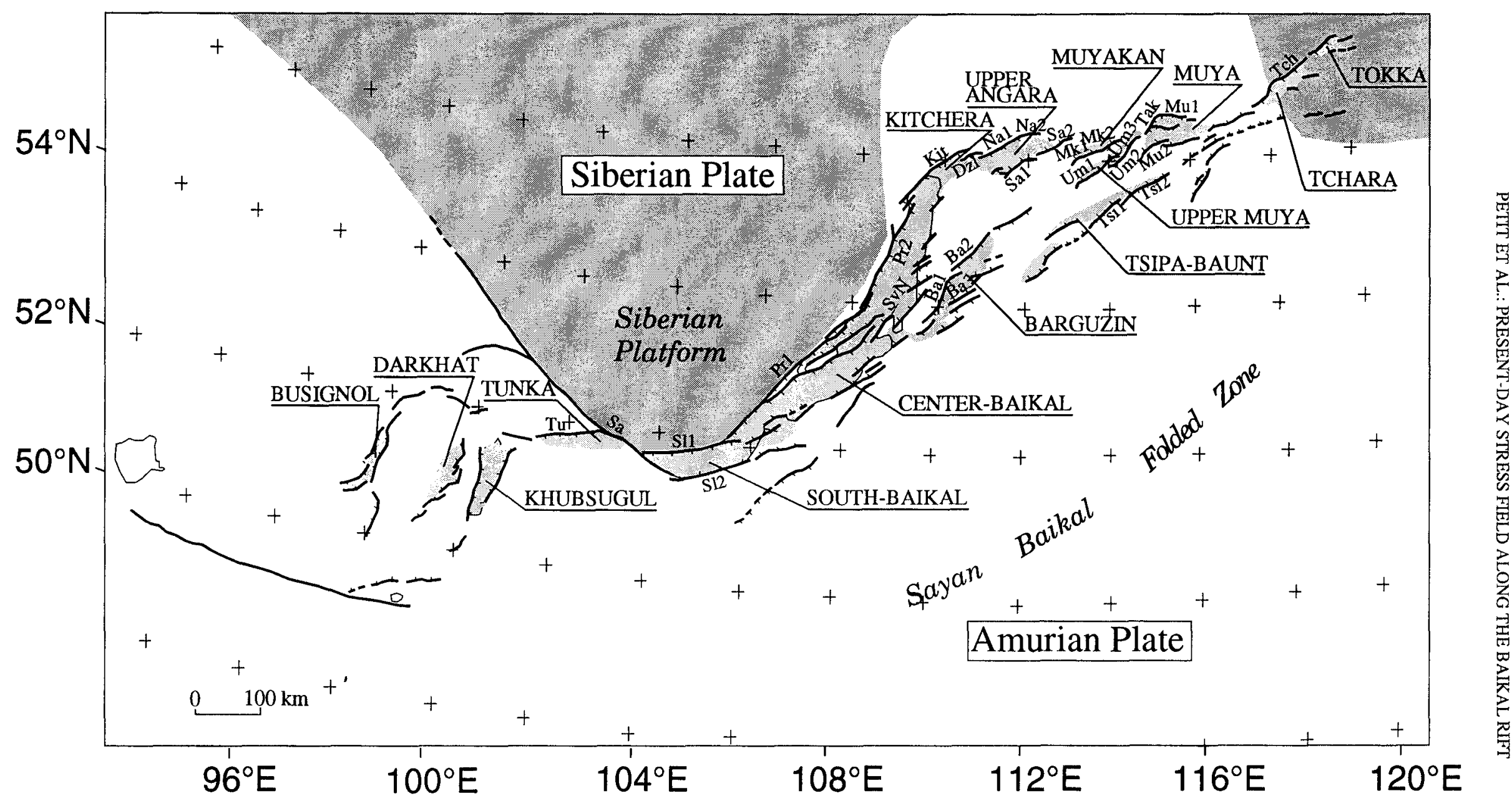

Figure 5. Simplified structural sketch of the Baikal rift area. Grey areas correspond to Siberian craton. Names of the basins are underlined. Small abreviations refer to faults used for striation prediction: Sa, Sayan; S11, Southern lake 1; S12, Southern lake 2; Pr1, Primorsky 1; Pr2, Primorsky 2; SvN, Svyatoy Nos; Ba1, Barguzin 1; Ba2, Barguzin 2; Ba3, Barguzin 3; Dzl, Dzelinda; Kit, Kitchera; Tsi1, Tsipa 1; Tsi2, Tsipa 2; Na1, North Angara 1; Na2, North Angara 2; Sa1, South Angara 1; Sa2, South Angara 2; Mk1, Muyakan 1; Mk2, Muyakan 2; Um1, Upper Muya 1; Um2, Upper Muya 2; Um3, Upper Muya 3; Mu1, Muya 1; Mu2, Muya 2; Tak, Taksimo; Tch, Tchara; Tu, Tunka [after Logatchev, 1993; Houdry, 1994]. 


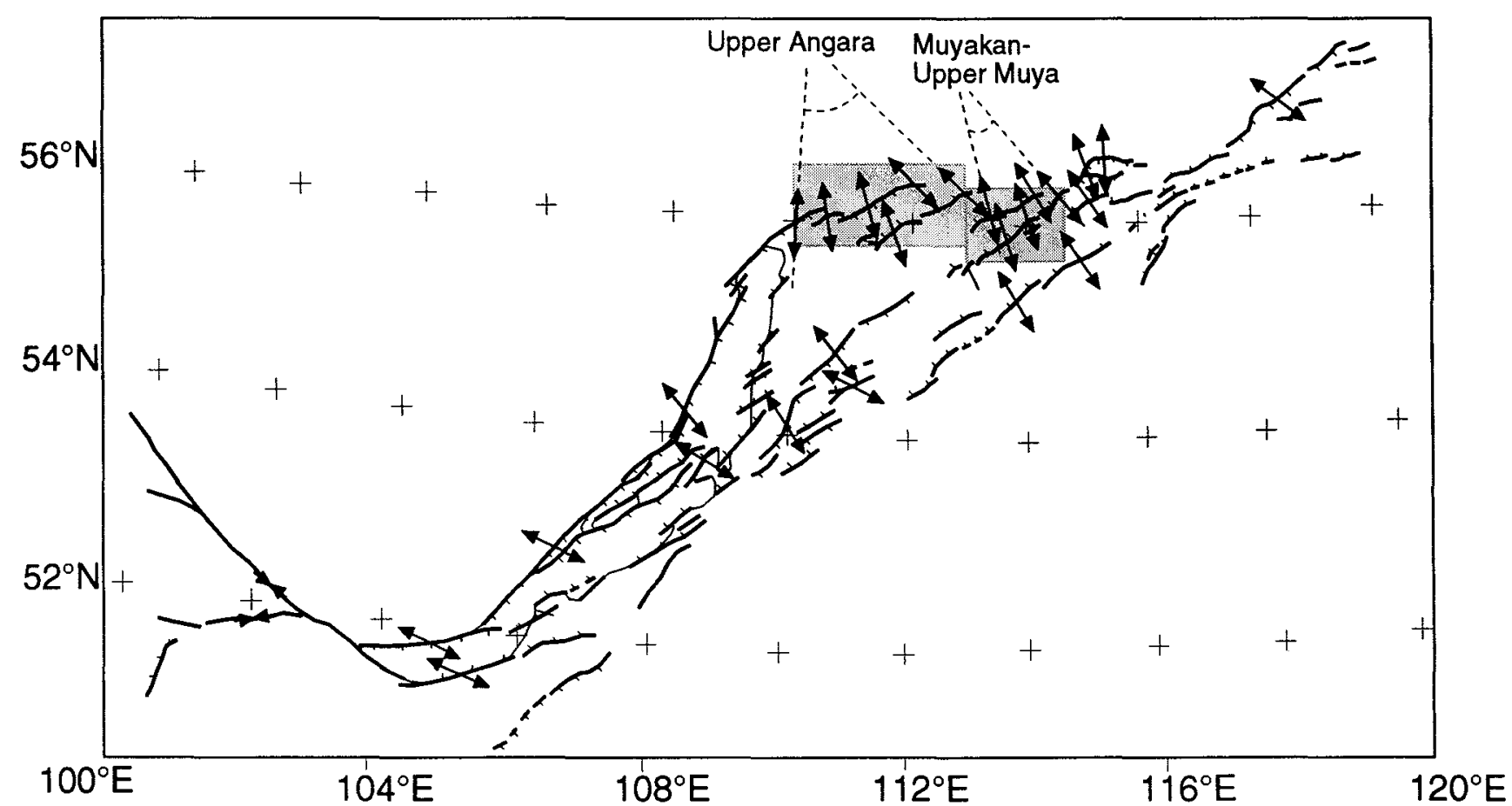

Figure 6. Map view of the 27 predicted slip vector azimuths (see Table 4 and text). Divergent and convergent arrows correspond to normal and reverse motions, respectively. Grey rectangles are the areas of counterclockwise slip vector rotations described in the text.

geometrical point of view, a rather satisfying explanation may be found when considering the particular shape of the hardly deformable Siberian shield bounding the SBR (Figure 5). In the westernmost part of the rift, if we assume an average $\mathrm{N} 40^{\circ} \mathrm{E}$ convergence vector between the Amurian and Siberian plates, then the NW-SE edge of the rigid platform may strongly resist the northeastward propagation of the compressional strain field. Consequently, reverse and sinistral faulting along the Sayan fault accommodates part of the convergence (Figure 9a). Actually, strike-slip faulting is predominant on the Sayan fault: it may be a result of a relative eastwards "escaping" of the Amurian plate. This kind of deformation is also predominant inside western Mongolia, especially along the major sinistral faults of Bolnai and Bogd [Baljinnyam et al., 1993]. The corner shape of the craton, at the southern tip of the Baikal lake, must play an important role in the accommodation of relative convergence motions: the Southern lake region (which is the deepest rift depression) is located against the SW-NE craton edge which is roughly parallel to the convergence direction. The opening of this part of the rift thus looks like a large-scale tension crack opening, where one border of the crack is resistant and the other one moves eastward (Figure 9b). Finally, the NBR is developing inside an indentation of the Siberian shield: most of the basins of the NBR are located in the Sayan-Baikal mobile belt, but both the large SW end and the sharp NE end of the NBR are bounded by the Siberian craton. We thus propose that, in the NBR too, the shieid geometry may have an indirect influence on the rift evolution, tending to resist the northeastward propagation of deformation (Figure 9c). This influence has already been noticed by Sherman [1978, 1992] and Logatchev [1993], who however point out that rift structures are more likely to propagate northward than southward. The rift zone seems more able to increase in width (i.e., inside the weakened folded zone) than in length (i.e., inside the resistant shield): this wide deformed zone could indicate large-scale extensional "cracking" of the lithosphere, as already suggested by Burov et al. [1994], inside which the opening gives rise to block rotations.

If these hypotheses are a possible way to explain observed motions in the Baikal rift, the overall three-dimensional behavior of the rift involves much more complex phenomena. For instance, the clear partitioning of the rift into two different zones especially suggests that anterift history may also strongly control the rheological behavior of the BRZ. Indeed, the rift is located on two main zones of weakened crust: the Paleozoic suture and the folded zone. The nature, as well as the dimension of these two weaknesses are quite different: one is a structural narrow boundary, the other one is a wide area of thickened crust, which thus offers a lower strength to extensional forces [Kusznir and Park, 1987]. When its orientation is favorable (i.e., in the SBR), the rift closely follows the suture between the Siberian craton and the Sayan-Baikal folded belt; when it becomes unfavorable (like in the NBR), it propagates inside the folded zone. The rheological contrast of these two deforming zones may thus also partly explain the location and varying geometry of the BRZ [Houdry, 1994]. Moreover, in the present study, we take the India-Asia collision as the only source of stress in the BRZ; we believe that it is an acceptable simplification for qualitative models like this one. However, two other factors must be taken into account to infer a quantitative model of deformation in this zone: first, the thermal effect resulting from possible lithospheric thinning beneath the Baikal region may create a weakening of the lithosphere [Lysak, 1992; Nicolas et al., 1994]; second, the whole 


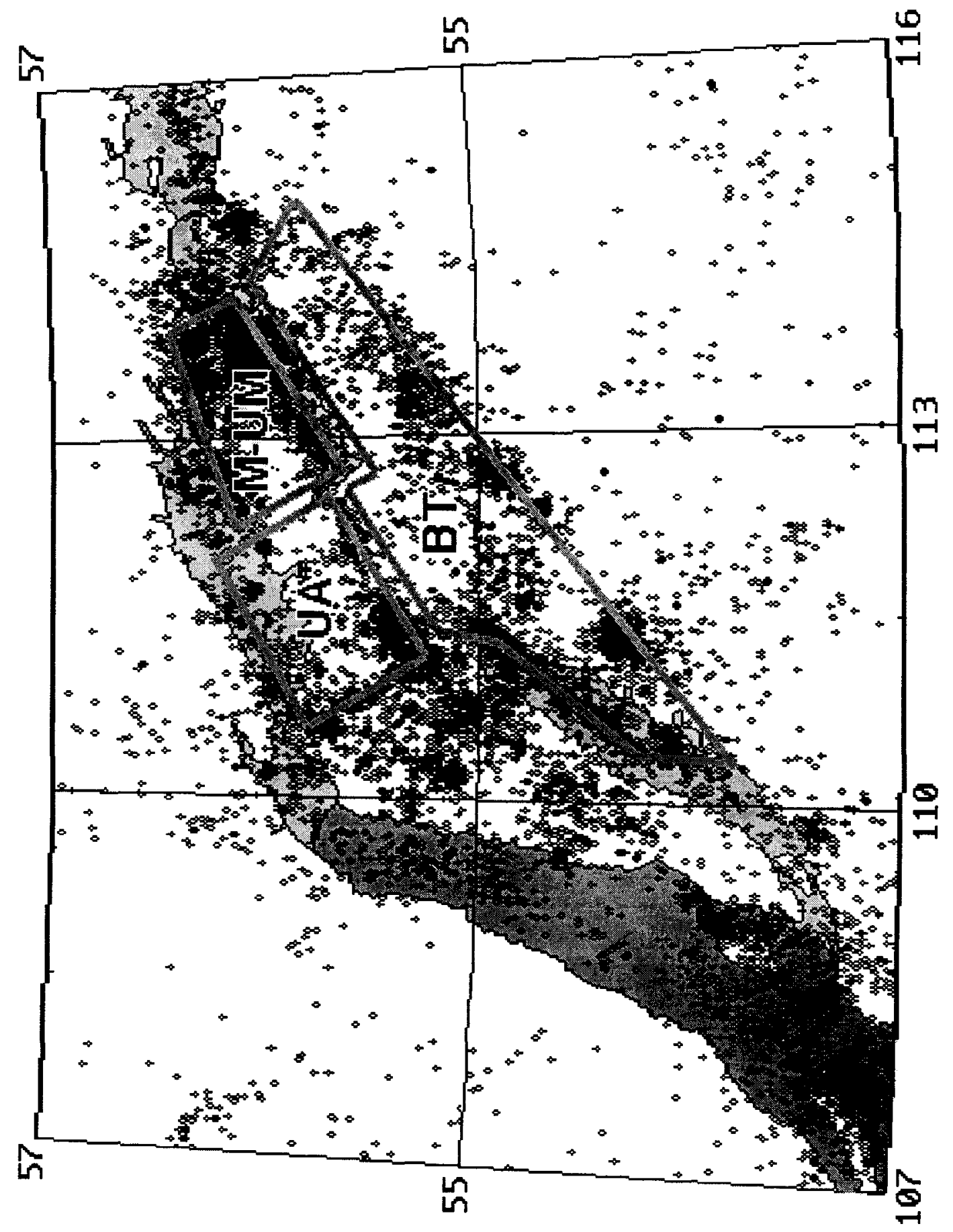

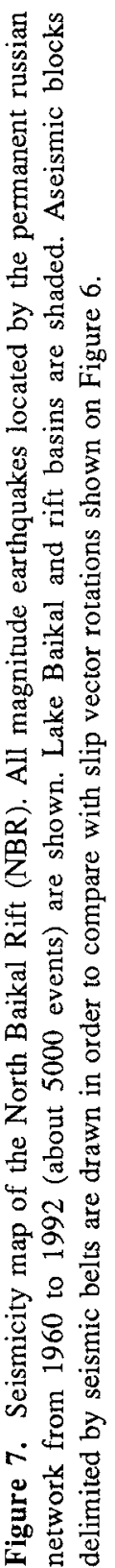




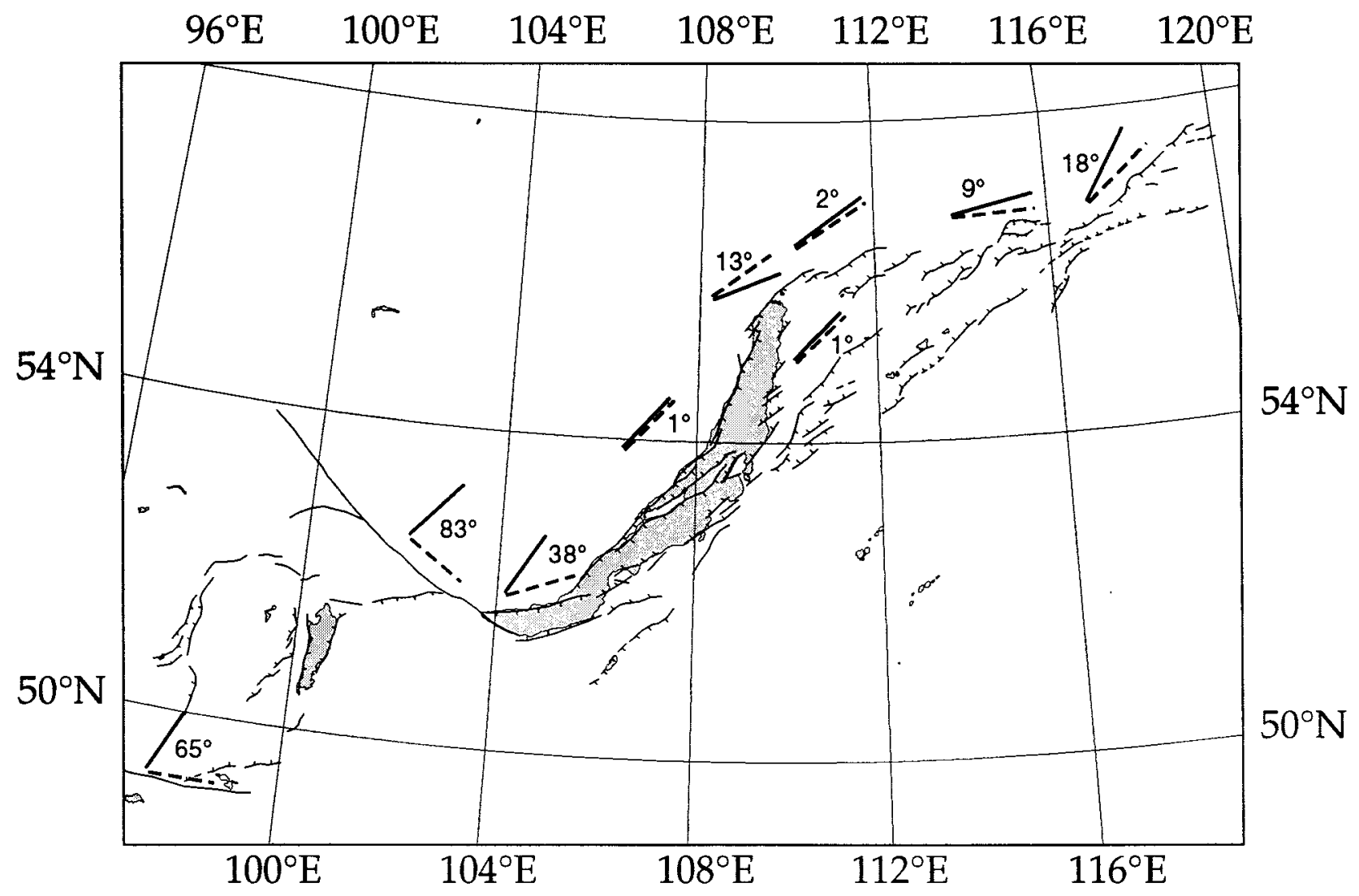

Figure 8. Map of angular differences between $S_{H \max }$ directions (thick lines) and active fault strikes (dashed lines), referred to the active fault pattern of the rift area (thin lines).

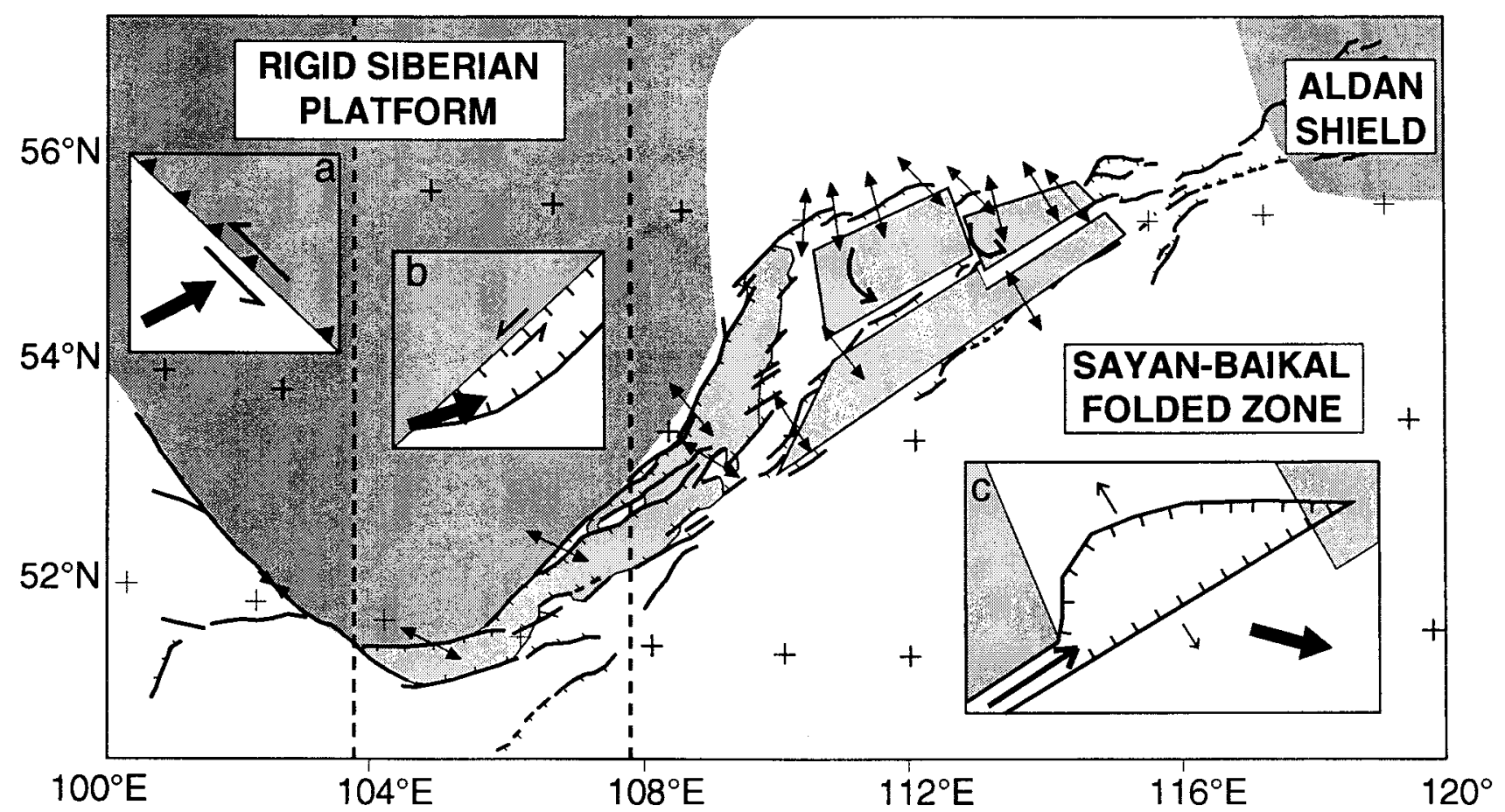

Figure 9. Schematic interpretation of the predicted motions in terms of rift deformation. Thick solid arrows in box a, box b, and box c are mean relative motions between Amurian and Siberian plates, extrapolated after Holt et al. [1995] and Houdry [1994]. Shaded areas are blocks depicted on Figure 7. Double arrows are slip vectors selected from Figure 6. See text for details. 
eastern border of the continent is bounded by subduction trenches whose tensional effect is not neglectable and can propagate far into the plate interior, contributing to its weakening [Whittaker et al., 1992]. Under these conditions, the forces resulting from the India-Asia collision, although strongly attenuated when reaching the remote Baikal region, can be important enough to produce a rift opening at this place. Consequently, the debate between passive and active rifting has a limited meaning here, as already mentioned elsewhere by Nicolas et al. [1994].
Acknowledgments. We dedicate this study to the memory of Alexandre V. Solonenko, who perished in the plane crash of January 1994 near Irkutsk. We thank O. Kuchai (Novosibirsk) for providing data in the Bolnai and Busingol areas. Comments and suggestions by Eric Calais were very appreciated, as well as constructive reviews by Evgueni Burov and Diane Doser. This work received support from NATO (grant CRG931637), CNRS-MDRI (1993-1995), CNRS-NSU ("Dynamique et Bilan de la Terre", and "Tectoscope-Positionnement") and the French Ministry of Foreign Affairs (Enveloppe "Echanges Scientifiques", 1993 and 1994). INSU-DBT ("Dynamique Globale") contribution $n^{\circ} 757$, and UMR "Géosciences Azur" contribution n5.

\section{References}

Angelier, J., Determination of the mean principal stresses for a given fault population, Tectonophysics, 56, 17-26, 1979.

Angelier, J, and P. Mechler, Sur une méthode graphique de recherche des contraintes principales également utilisable en tectonique et en sismologie: La méthode des dièdres droits, Bull. Soc. Géol. Fr., XIX, 651-652, 1977.

Avouac, J.-P., and P. Tapponnier, Kinematic model of deformation in central Asia, Geophys. Res. Lett., 20, 895-898, 1993.

Baljinnyam, I., A. Bayasgalan, B. A. Borisov, A. Cisternas, M. G. Dem yanovich, L. Ganbaatar, V. M. Kochetkov, R. A. Kurushin, P. Molnar, H. Philip, and Y. Y. Vashchilov, Ruptures of major earthquakes and active deformation in Mongolia and its surroundings, 62 pp., Geol. Soc. Am. Mem. 181, 1993

Balla, Z., M. Kuzmin, and K. Levi, Kinematics of the Baikal opening: Results of modelling, Ann. Tectonic., 1, 18-31, 1991.

Bosworth, W., M. R. Strecker, and P. M. Blisniuk, Integration of east African paleostress and present-day stress data: Implication for continental stress field dynamics, J. Geophys. Res., 97, 11,851$11,865,1992$.

Burov, E. B., F. Houdry, M. Diament, and J. Déverchère, $A$ broken plate beneath the Baikal rift zone revealed by gravity modelling, Geophys. Res. Lett., 21, 129. $132,1994$.

Carey-Gailhardis, E., and J.-L. Mercier, A numerical method for determining the state of stress using focal mechanisms of earthquake populations: Application to Tibetan teleseisms and microseismicity of southern Peru, Earth Planet. Sci. Lett., 82, 165-179, 1987.

Comet, F. H., and D. Burlet, Stress field determination in France by hydraulic tests in boreholes, J. Geophys. Res., 97, 11,829$11,850,1992$

Davy, P., and P. R. Cobbold, Indentation tectonics in nature and experiment, 1, Experiments scaled for gravity, Bull. Geol. Inst. Univ. Uppsala, N.S. 14, 129-141, 1988.
Delvaux, D., A. Melnikov, and V. Ermikov, Paleostress reconstruction and geodynamics of the Baikal region, Central Asia, I, Paleozoic and Mesozoic pre-rift evolution Tectonophysics, 252, 61-101, 1996.

Déverchère, J., F. Houdry, M. Diament, N. V. Solonenko, and A. V. Solonenko, Evidence for a seismogenic upper mantle and lower crust in the Baikal rift, Geophys. Res. Lett., 18, 1099-1102, 1991

Déverchère, J., F. Houdry, N. V. Solonenko, A. V. Solonenko, and V. A. Sankov, Seismicity, active faults and stress field of the North Muya region, Baikal rift: New insights on the rheology of extended continental lithosphere, J. Geophys. Res., 98, 19,895-19,912, 1993.

Diament, M., and M. G. Kogan, Long wavelength gravity anomalies and the deep thermal structure of the Baikal rift, Geophys. Res. Lett., 17, 1977-1980, 1990.

Doser, D. I., Faulting within the westem Baikal rift as characterized by earthquake studies, Tectonophysics, 196, 87-107, 1991a.

Doser, D. I., Fauling within the eastem Baikal rift as characterized by earthquake studies, Tectonophysics, 196, 109-139, $1991 \mathrm{~b}$.

Dziewonski, A. M., and J. H. Woodhouse, An experiment in the systematic study of global seismicity: Centroid-moment tensor solutions for 201 moderate and large earthquakes of 1981, J. Geophys. Res., 88, 3247-3271, 1983.

Ermikov, V. D., Mesozoic precursors of the Cenozoic rift structures of Central Asia, Bull. Centres Rech. Explor.Prod. Elf Aquitaine, 18, 123-134, 1994.

Gao, S., P. M. Davis, H. Liu, P. D. Slack, Y. A. Zorin, N. A. Logatchev, M. G. Kogan, P. D. Burkholder, and R. P. Meyer, Asymmetric upwarp of the asthenosphere beneath the Baikal rift zone, Siberia, J. Geophys. Res., 99, 15,319-15,330, 1994.

Gephart, J. W., and D. W. Forsyth, An improved method for determining the regional stress tensor using earthquake focal mechanism data: Application to the San Fernando earthquake sequence, $J$. Geophys Res., 89, 9305-9320, 1984

Golenetsky, S. I., Problems of the seismicity of the Baikal rift zone, J. Geodyn., 11, 293 $307,1990$.
Hartse, H. E., M. C. Fehler, R. C. Aster, J. S. Scott, and F. L. Vernon, Small-scale stress heterogeneity in the Anza seismic gap, southern California, J. Geophys. Res., 99, 6801-6818, 1994.

Hippolyte, J.-C., J. Angelier, and F. Roure, A major geodynamic change revealed by Quaternary stress pattems in the Southern Apennines (Italy), Tectonophysics, 230, 199-210, 1994.

Holt, W. E., M. Li, and A. J. Haines, Earthquake strain rates and instantaneous relative motions within central and eastern Asia, Geophys. J.Int., 122, 569-593, 1995.

Horiuchi, S., G. Rocco, and A. Hasegawa, Discrimination of fault planes from auxiliary planes based on simultaneous determination of stress tensor and large number of fault plane solutions, J. Geophys. Res., 100, 8327-8338, 1995.

Houdry, F., Mécanismes de l'extension continentale dans le rift Nord Baikal, Sibérie, 356 pp., Thèse de Doctorat, Univ. Pierre et Marie Curie (Paris VI), 1994.

Houseman, G., and P. England, Crustal thickening versus lateral expulsion in the India-Asian continental collision, $J$. Geophys. Res., 98, 12,233-12,249, 1993.

Kusznir, N. J. and R. G. Park, 1987, The extensional strength of the continental lithosphere: Its dependence on geothermal gradient, and crustal composition and thickness, in Continental Extensional Tectonics, Geol. Soc. Spec. Publ., M. P. Coward, J. F. Dewey and P. L. Hancock cds, 35-52.

Logatchev, N. A., History and geodynamics of the Lake Baikal Rift in the context of the Eastem Siberia rift system: A review, Bull Centres Rech. Explor.-Prod. Elf Aquitaine, 17, 353-370, 1993.

Lysak, S. V., Heal flow variations in continental rifts, Tectonophysics, 208, 309. 323, 1992.

McCalpin, J. P., and V. S. Khromovskikh, Holocene paleoseismicity of the Tunka fault, Baikal rift, Russia, Tectonics, 14, 594 $605,1995$.

McKenzie, D. M., The relation between fault plane solutions for earthquakes and the direction of the principal stresses, Bull. Seismol. Soc. Am., 59, 591-601, 1969. 
Michael, A. J., Use of focal mechanisms to determine stress: A control study, $J$ Geophys. Res., 92, 357-368, 1987.

Molnar, P., and Q. Deng, Faulting associaled with large earthquakes and the average rate of deformation in central and eastem Asia, J. Geophys. Res., 89, 6203-6227, 1984

Molnar, P., and P. Tapponnier, Cenozoic tectonics of Asia: Effects of a continental collision, Science, 189, 419-426, 1975.

Nicolas, A., U. Achauer, and M. Daignières, Rift initiation by lithospheric rupture, Earth Planet. Sci. Lett., 123, 281-298, 1994.

Peltzer, G., and P. Tapponnier, Formation and evolution of strike-slip faults, rifts and basins during the India-Asia collision: An experimental approach, J. Geophys. Res., 93, 15,095-15,117, 1988.

Petil, C., Analyse de séquences sismiques par relocalisation: Application au rift NordBaikal et interprétation tectonique, $57 \mathrm{pp}$., Rapp. de DEA de l'Univ. Pierre et Marie Curie (Paris VI), 1993.

Petit, C., and J. Déverchère, Velocity structure of the northern Baikal rift, Siberia, from local and regional earthquake travel times, Geophys. Res. Let., 22, 1677-1680, 1995.

Petil, C., J. Déverchère, E. Calais, A. Deschamps, K. G. Levi, V. A. Sankov, V. V. Ruzhich, A. I. Miroshnichenko, N. V. Solonenko, and O. Petukhov, Active tectonics, stress tensor analysis, and firsi GPS survey of the southern Baikal rift, EOS Trans. $A G U$, Fall Meet. suppl., 75 (44), 181, 1994.

Rebai, S., H. Philip, and A. Taboada, Modern tectonic stress field in the Mediterranean region: Evidence for variation in stress directions at different scales, Geophys. $J$. Int., 110, 106-140, 1992.

Richardson, R. M., Ridge forces, absolute plate motions, and the intraplate stress field, $J$. Geophys. Res., 97, 11,739-11,748, 1992.

Ritz, J.-F., Determining the slip vector by graphical construction: Use of a simplified representation of the stress tensor, J. Struct. Geol., 16, 737-741, 1994.

Rivera, L., and A. Cistemas, Stress tensor and fault plane solutions for a population of earthquakes, Bull. Seismol. Soc. Am., 80, $600-614,1990$

Ruppel, C. D., M. G. Kogan, and M. K. McNutt, Implications of new gravity data for Baikal rift zone structure, Geophys. Res. Lett., 20, 1635-1638, 1993.

Shamir, G., M. D. Zoback, and C. B. Barton, In situ stress orientation near the San Andreas fault: Preliminary results to $2.1 \mathrm{~km}$ depth from the Cajon Pass scientific drillhole, Geophys. Res. Letl., 15, 989-992, 1988.

Shemnan, S. I., Faults of the Baikal rift zone, Tectonophysics, 45, 31-39, 1978.

Sheman, S. I., Faults and tectonic stresses of the Baikal rift zone, Tectonophysics, 208, 297-307, 1992

Solonenko, A. V., N. V. Solonenko, V. I. Melnikova, B. M. Kuzmin, O. A. Kuchai, and S. S. Sukhanova, Stresses and fault plane motions of earthquakes in Siberia and Mongolia (in Russian), Seismicity and seismic zoning of Northern Eurasia, IFE RAS, 1, 113-122, 1993.

Solonenko, N. V., The energy released in seismic sequences of the Baikal rift zone, Proc. 3rd International Symposium on the analysis of seismicity and seismic risk, Liblice Castle, Prag, Czechoslovakia, 216224, 1985.

Solonenko, V. P., Seismic regional division of eastern Siberia and its geologicalgeophysical base (in Russian), Nauka, Novosibirsk, 92-162, 1977.

Tapponnier, P., and P. Molnar, Active faulting and Cenozoic tectonics of the Tien Shan, Mongolia and Baykal regions, J. Geophys. Res., 84, 3425-3459, 1979

Tapponnier, P., G. Peltzer, A. Y. Le Dain, R. Armijo, and P. R. Cobbold, Propagating extrusion tectonics in Asia: New insights from simple experiments with plasticine, Geology, 10, 611-616, 1982.
Whittaker, A., M. H. P. Bott, and G. D. Waghom, Stresses at plate boundary forces associated with subduction plate margins, $J$. Geophys. Res., 97, 11,933-11,944, 1992.

Xu, Z., W. Suyun, H. Yurui, and G. Ajia, Tectonic stress field of China inferred from a large number of small earthquakes, $J$. Geophys. Res., 97, 11,867-11,878, 1992.

Zamarayev, S. M., and V. V. Ruzhich, On relationships between the Baikal and ancien structures, Tecionophysics, 45, 4147, 1978.

Zoback, M. L., First-and second-order patterns of stress in the lithosphere: The World Stress Map Project, J. Geophys. Res., 97, 11,703-11,728, 1992.

Zoback, M. D., and G. C. Beroza Heterogeneous slip and siress release in the Loma Prieta earthquake, II, Evidence for near frictionless faulting and complete coseismic stress drop, EOS Trans. AGU, Fall Meeting suppl., 72 (44), 309, 1991.

Zoback, M. L., et al., Global patterns of tectonic stresses, Nature, 341, 291-298, 1989

Zonenshain, L. P., and L. A. Savostin, Geodynamics of the Baikal rift zone and plate tectonics of Asia, Tectonophysics, 76 $1-45,1980$.

J. Déverchère, F. Houdry, and C. Petit, Laboratoire de Géodynamique Sous-Marine, Observatoire Océanologique, La Darse, BP48, 06230 Villefranche-sur-Mer, France. (e-mail: jack@ccrv.obs.vlfr.fr)

V. I. Melnikova, V. A. Sankov, Institute of the Earth's Crust, Lemontov St. 128, Irkutsk 664033, Russia. (e-mail: san@cora.irkutsk.su)

D. Delvaux, Royal Museum for Central Africa, Leuvensesteenweg, 13, B-3080 Tervuren, Belgium. (e-mail: ddelvaux@vub.ac.be)

(Received October 20, 1995 revised January 22, 1996; accepted January 22, 1996.) 